\title{
SOLUTIONS WITH SIGN INFORMATION FOR NONLINEAR NONHOMOGENEOUS ELLIPTIC EQUATIONS
}

\author{
Nikolaos S. Papageorgiou — Vicenţiu D. RĂdulescu
}

\begin{abstract}
We consider a class of nonlinear, coercive elliptic equations driven by a nonhomogeneous differential operator. Using variational methods together with truncation and comparison techniques, we show that the problem has at least three nontrivial solutions, all with sign information. In the special case of $(p, 2)$-equations, using tools from Morse theory, we show the existence of four nontrivial solutions, all with sign information. Finally, for a special class of parametric equations, we obtain multiplicity theorems that substantially extend earlier results on the subject.
\end{abstract}

\section{Introduction}

Let $\Omega \subseteq \mathbb{R}^{N}$ be a bounded domain with a $C^{2}$-boundary $\partial \Omega$. In this paper we study the existence of multiple nontrivial solutions for the following nonlinear Dirichlet problem:

$$
-\operatorname{div} a(D u(z))=f(z, u(z)) \quad \text { in } \Omega,\left.\quad u\right|_{\partial \Omega}=0 .
$$

The map $a: \mathbb{R}^{N} \rightarrow \mathbb{R}^{N}$ involved in the differential operator of (1.1) is strictly monotone and satisfies certain other regularity conditions listed in hypotheses $\mathrm{H}(\mathrm{a})$ below.

2010 Mathematics Subject Classification. 35J20, 35J60, 35J92, 58E05.

Key words and phrases. Nonlinear nonhomogeneous differential operator, nonlinear maximum principle, strong comparison principle, Morse theory, parametric equations, nodal solutions. 
Special cases of the differential operator in (1.1), are the $p$-Laplacian, the $(p, q)$-Laplace operator (the sum of a $p$-Laplacian and of a $q$-Laplacian), and the generalized $p$-mean curvature differential operator. It is important to notice that the differential operator in (1.1) is not necessarily homogeneous. The reaction term $f(z, x)$ is a Carathéodory function (that is, for all $x \in \mathbb{R}$, the mapping $z \mapsto f(z, x)$ is measurable and for almost all $z \in \Omega$, the map $x \mapsto f(z, x)$ is continuous). Our hypotheses on the growth of $f(z, \cdot)$ ensure that the energy functional of the problem is coercive.

We prove a "three solutions theorem" providing sign information for all the solutions produced. In the particular case where $a(y)=\|y\|^{p-2} y+y$ with $2 \leq p<\infty$ (the differential operator is the sum of a $p$-Laplacian and the Laplace operator), we show that we can have four nontrivial solutions, all with sign information (one positive, one negative, and two nodal (that is, sign changing)).

Multiplicity results for coercive elliptic problems were proved for semilinear equations driven by the Laplace operator by Ambrosetti and Lupo [1], Ambrosetti and Mancini [2], and Struwe [29], [30]. For equations driven by the $p$-Laplacian, we refer to the works by Liu [19], Liu and Liu [20], and Papageorgiou and Papageorgiou [24]. None of the aforementioned works produces nodal solutions.

Our approach is variational and it is based on the critical point theory in combination with suitable truncation and comparison techniques and Morse theory. We show that problem (1.1) has at least three nontrivial smooth solutions, one positive, one negative, and a third nodal. In the particular case when the differential operator is the sum of a $p$-Laplacian $(p \geq 2)$ and a Laplacian, we show that we can have a second nodal solution for a total of four nontrivial smooth solutions, all with sign information. Finally, we show that for a special case of parametric nonlinear equations driven by the $p$-Laplacian, our multiplicity theorems lead to a substantial improvement of the results of Ambrosetti and Lupo [1], Ambrosetti and Mancini [2], Struwe [29], [30], and Papageorgiou and Papageorgiou [24].

\section{Mathematical background and auxiliary results}

Let $X$ be a Banach space and $X^{*}$ be its topological dual. By $\langle\cdot, \cdot\rangle$ we denote the duality brackets for the pair $\left(X^{*}, X\right)$. Given $\varphi \in C^{1}(X)$, we say that $\varphi$ satisfies the "Palais-Smale" condition (the "PS-condition" for short), if the following is true:

"Every sequence $\left\{x_{n}\right\}_{n \geq 1} \subseteq X$ such that $\left\{\varphi\left(x_{n}\right)\right\}_{n \geq 1} \subseteq \mathbb{R}$ is bounded and $\varphi^{\prime}\left(x_{n}\right) \rightarrow 0$ in $X^{*}$ as $n \rightarrow \infty$, admits a strongly convergent subsequence".

This compactness-type condition which compensates for the fact that the ambient space $X$ need not be locally compact, leads to a deformation theorem 
for the functional $\varphi$, from which we can derive the minimax theory of certain critical values of $\varphi$. One such minimax theorem is the so-called "mountain pass theorem", which we recall here for future use. For details we refer to Gasinski and Papageorgiou [13], Kristaly, Rădulescu and Varga [16], and Rădulescu [28].

Theorem 2.1. Assume that $\varphi \in C^{1}(X)$ satisfies the PS-condition, $x_{0}, x_{1} \in$ $X,\left\|x_{1}-x_{0}\right\|>\rho>0$,

$$
\max \left\{\varphi\left(x_{0}\right), \varphi\left(x_{1}\right)\right\}<\inf \left\{\varphi(x):\left\|x-x_{0}\right\|=\rho\right\}=\eta_{\rho}
$$

and $c=\inf _{\gamma \in \Gamma \in[0,1]} \max _{t \in[} \varphi(\gamma(t))$, where $\Gamma=\left\{\gamma \in C([0,1], X): \varphi(0)=x_{0}, \varphi(1)=x_{1}\right\}$.

Then $c \geq \eta_{\rho}$ and $c$ is a critical value of $\varphi$.

The analysis of problem (1.1) will use the Sobolev space $W_{0}^{1, p}(\Omega)$ and the Banach space

$$
C_{0}^{1}(\bar{\Omega})=\left\{u \in C^{1}(\bar{\Omega}): u=0 \text { on } \partial \Omega\right\} .
$$

The latter is an ordered Banach space with positive cone given by

$$
C_{+}=\left\{u \in C_{0}^{1}(\bar{\Omega}): u(z) \geq 0 \text { for all } z \in \bar{\Omega}\right\} .
$$

This cone has a nonempty interior given by

$$
\operatorname{int} C_{+}=\left\{u \in C_{+}: u(z)>0 \text { for all } z \in \Omega, \frac{\partial u}{\partial n}(z)<0 \text { for all } z \in \partial \Omega\right\},
$$

where $n(\cdot)$ is the outward unit normal on $\partial \Omega$.

Let $\vartheta \in C^{1}(0, \infty)$ and assume that

$$
\begin{aligned}
0<\widehat{c} \leq \frac{t \vartheta^{\prime}(t)}{\vartheta(t)} \leq c_{0} & \text { for all } t>0 \text { and some } c_{0}, \widehat{c}>0 ; \\
c_{1} t^{p-1} \leq \vartheta(t) \leq c_{2}\left(1+t^{p-1}\right) & \text { for all } t>0 \\
& \text { and some } c_{1}, c_{2}>0,1<p<\infty .
\end{aligned}
$$

Our hypotheses on the map $y \mapsto a(y)$ are the following:

$\mathrm{H}(\mathrm{a}) a(y)=a_{0}(\|y\|) y$ for all $y \in \mathbb{R}^{N}$, with $a_{0}(t)>0$ for all $t>0$ and

(i) $a_{0} \in C^{1}(0, \infty), t \mapsto t a_{0}(t)$ is strictly increasing in $(0, \infty), t a_{0}(t) \rightarrow 0$ as $t \rightarrow 0^{+}$and

$$
\lim _{t \rightarrow 0^{+}} \frac{t a_{0}^{\prime}(t)}{a_{0}(t)}=c>-1
$$

(ii) for every $y \in \mathbb{R}^{N} \backslash\{0\}$, we have

$$
\|\nabla a(y)\| \leq c_{3} \frac{\vartheta(\|y\|)}{\|y\|} \text { for some } c_{3}>0 ;
$$

(iii) for every $y \in \mathbb{R}^{N} \backslash\{0\}$, we have

$$
(\nabla a(y) \xi, \xi)_{\mathbb{R}^{N}} \geq \frac{\vartheta(\|y\|)}{\|y\|}\|\xi\|^{2} \quad \text { for all } \xi \in \mathbb{R}^{N} ;
$$


(iv) if $G_{0}(t)=\int_{0}^{t} s a_{0}(s) d s$ for all $t>0$, then there exists $\tau \in(1, p]$ such that $t \mapsto G_{0}\left(t^{1 / \tau}\right)$ is convex in $(0,+\infty)$ and $\lim _{t \rightarrow 0^{+}} \tau G_{0}(t) / t^{\tau}=\widetilde{c}>0$.

REMARK 2.2. The above assumptions show that the function $G_{0}(\cdot)$ is strictly convex and strictly increasing. We set $G(y)=G_{0}(\|y\|)$ for all $y \in \mathbb{R}^{N}$. Then $G(\cdot)$ is convex, $G(0)=0$ and

$$
\nabla G(y)=G_{0}^{\prime}(\|y\|) \frac{y}{\|y\|}=a_{0}(\|y\|) y=a(y) \quad \text { for all } y \in \mathbb{R}^{N} \backslash\{0\} .
$$

Therefore $G(\cdot)$ is the primitive of $a(\cdot)$. The convexity of $G(\cdot)$ and the fact that $G(0)=0$ imply that

$$
G(y) \leq(a(y), y)_{\mathbb{R}^{N}} \quad \text { for all } y \in \mathbb{R}^{N} .
$$

Using hypotheses $\mathrm{H}(\mathrm{a})$ and (2.1), (2.2), we deduce the following lemma summarizing the properties of $a(\cdot)$.

Lemma 2.3. Assume that hypotheses $\mathrm{H}(\mathrm{a})$ hold. Then the following properties hold:

(a) the operator $y \mapsto a(y)$ is maximal monotone and strictly monotone;

(b) $\|a(y)\| \leq c_{4}\left(1+\|y\|^{p-1}\right)$ for all $y \in \mathbb{R}^{N}$ and some $c_{4}>0$;

(c) $(a(y), y)_{\mathbb{R}^{N}} \geq c_{1}\|y\|^{p} /(p-1)$ for all $y \in \mathbb{R}^{N}$.

Using this lemma and the integral form of the mean value theorem, we obtain the following growth conditions for the primitive $G(\cdot)$.

Corollary 2.4. Assume that hypotheses $\mathrm{H}(\mathrm{a})$ hold. Then

$$
\frac{c_{1}}{p(p-1)}\|y\|^{p} \leq G(y) \leq c_{5}\left(1+\|y\|^{p}\right) \quad \text { for all } y \in \mathbb{R}^{N} \text { and some } c_{5}>0 \text {. }
$$

EXAMPLES 2.5. The following maps satisfy hypotheses $\mathrm{H}(\mathrm{a})$ :

(a) $a(y)=\|y\|^{p-2} y$ with $1<p<\infty$. Then the corresponding differential operator is the $p$-Laplacian $\Delta_{p} u=\operatorname{div}\left(\|D u\|^{p-2} D u\right)$ for all $u \in W_{0}^{1, p}(\Omega)$. This quasilinear operator arises in many applications such as non-Newtonian fluid flows and turbulent filtration in porous media.

(b) $a(y)=\|y\|^{p-2} y+\mu\|y\|^{q-2} y$ with $1<q<p, p \geq 2$ and $\mu \geq 0$. Then the corresponding differential operator is the $(p, q)$-differential operator

$$
u \mapsto \Delta_{p} u+\mu \Delta_{q} u \text { for all } u \in W_{0}^{1, p}(\Omega) .
$$

Equations driven by such differential operators are important in mathematical physics. We refer to Benci, D'Avenia, Fortunato and Pisani [5] (existence of solitons for problems of quantum physics) and Cherfils and Ilyasov [7] (problems in plasma physics). Recently, multiplicity results for resonant equations of this type were proved by Papageorgiou and Rădulescu [25]. 
(c) $a(y)=\left(1+\|y\|^{2}\right)^{(p-2) / 2} y$ with $1<p<\infty$. Then the corresponding differential operator is the generalized $p$-mean curvature differential operator

$$
u \mapsto \operatorname{div}\left(\left(1+\|D u\|^{2}\right)^{(p-2) / 2} D u\right) \quad \text { for all } u \in W_{0}^{1, p}(\Omega) .
$$

(d) $a(y)=\|y\|^{p-2} y+\|y\|^{p-2} y /\left(1+\|y\|^{p}\right)$ with $1<p<\infty$.

Let $A: W_{0}^{1, p}(\Omega) \rightarrow W^{-1, p^{\prime}}(\Omega)=W_{0}^{1, p}(\Omega)^{*}\left(1 / p+1 / p^{\prime}=1\right)$ be the nonlinear map defined by

$$
\langle A(u), y\rangle=\int_{\Omega}(a(D u(z)), D y(z))_{\mathbb{R}^{N}} d z \quad \text { for all } u, y \in W_{0}^{1, p}(\Omega) .
$$

According to Gasinski and Papageorgiou [14], we have:

Proposition 2.6. Assume that hypotheses $\mathrm{H}(\mathrm{a})$ hold. Then the operator $A: W_{0}^{1, p}(\Omega) \rightarrow W^{-1, p^{\prime}}(\Omega)$ defined by $(2.3)$ is bounded (that is, maps bounded sets to bounded sets), continuous, maximal monotone, and of type $(\mathrm{S})_{+}$, that is, if $u_{n} \stackrel{w}{\longrightarrow} u$ in $W_{0}^{1, p}(\Omega)$ and $\lim _{n \rightarrow \infty}\left\langle A\left(u_{n}\right), u_{n}-u\right\rangle \leq 0$, then $u_{n} \rightarrow u$ in $W_{0}^{1, p}(\Omega)$.

Let $f_{0}: \Omega \times \mathbb{R} \rightarrow \mathbb{R}$ be a Carathéodory function with subcritical growth in the $x \in \mathbb{R}$ variable, that is,

$$
\left|f_{0}(z, x)\right| \leq a(z)\left(1+|x|^{r-1}\right) \quad \text { for a.a. } z \in \Omega \text { and all } x \in \mathbb{R},
$$

with $a \in L^{\infty}(\Omega)_{+}$and

$$
1<r<p^{*}= \begin{cases}\frac{N p}{N-p} & \text { if } p<N, \\ +\infty & \text { if } p \geq N .\end{cases}
$$

We set $F_{0}(z, x)=\int_{0}^{x} f_{0}(z, s) d s$ and consider the $C^{1}$-functional $\varphi_{0}: W_{0}^{1, p}(\Omega)$ $\rightarrow \mathbb{R}$ defined by

$$
\varphi_{0}(u)=\int_{\Omega} G(D u(z)) d z-\int_{\Omega} F_{0}(z, u(z)) d z \quad \text { for all } u \in W_{0}^{1, p}(\Omega) .
$$

The next result is concerned with $C_{0}^{1}(\bar{\Omega})$ and $W_{0}^{1, p}(\Omega)$ local minimizers of $\varphi_{0}$. The first such property was proved by Brezis and Nirenberg [6] when $G(y)=$ $\|y\|^{2} / 2$ and it was extended by Garcia Azorero, Manfredi and Peral Alonso [12] to the case $G(y)=\|y\|^{p} / p, 1<p<\infty$ (see also Guo and Zhang [15] where $2 \leq$ $p<\infty)$. The following result can be found in Gasinski and Papageorgiou [14]. In the sequel we denote by $\|\cdot\|$ the norm in the Sobolev space $W_{0}^{1, p}(\Omega)$. By virtue of the Poincaré inequality, $\|u\|=\|D u\|_{p}$ for all $u \in W_{0}^{1, p}(\Omega)$.

Proposition 2.7. Let hypotheses $\mathrm{H}(\mathrm{a})(\mathrm{i})$-(iii) hold and $u_{0} \in W_{0}^{1, p}(\Omega)$ is a local $C_{0}^{1}(\bar{\Omega})$-minimizer of $\varphi_{0}$, that is, there exists $\rho_{0}>0$ such that

$$
\varphi_{0}\left(u_{0}\right) \leq \varphi_{0}\left(u_{0}+h\right) \quad \text { for all } h \in C_{0}^{1}(\bar{\Omega}) \text { with }\|h\|_{C_{0}^{1}(\bar{\Omega})} \leq \rho_{0} .
$$


Then $u_{0} \in C_{0}^{1, \beta}(\bar{\Omega})$ with $\beta \in(0,1)$ and $u_{0}$ is a local $W_{0}^{1, p}(\Omega)$-minimizer of $\varphi_{0}$, that is, there exists $\rho_{1}>0$ such that

$$
\varphi_{0}\left(u_{0}\right) \leq \varphi_{0}\left(u_{0}+h\right) \quad \text { for all } h \in W_{0}^{1, p}(\Omega) \text { with }\|h\| \leq \rho_{1} .
$$

For $h_{1}, h_{2} \in L^{\infty}(\Omega)$, we write $h_{1} \prec h_{2}$ if for every compact set $K \subseteq \Omega$ there exists $\varepsilon=\varepsilon(K)>0$ such that

$$
h_{1}(z)+\varepsilon \leq h_{2}(z) \quad \text { for a.a. } z \in K .
$$

Evidently, if $h_{1}, h_{2} \in C(\Omega)$ and $h_{1}(z)<h_{2}(z)$ for all $z \in \Omega$, then $h_{1} \prec h_{2}$.

From Papageorgiou and Rădulescu [25], we recall the following strong comparison principle (see also Arcoya and Ruiz [3, Proposition 2.6]).

Proposition 2.8. Assume $\xi \geq 0, h_{1}, h_{2} \in L^{\infty}(\Omega), h_{1} \prec h_{2}$, and $u_{1}, u_{2} \in$ $C_{0}^{1}(\bar{\Omega})$ with $u_{2} \in \operatorname{int} C_{+}$are solutions of

$$
\begin{aligned}
& -\Delta_{p} u_{1}(z)-\Delta u_{1}(z)+\xi\left|u_{1}(z)\right|^{p-2} u_{1}(z)=h_{1}(z) \text { in } \Omega \text {, } \\
& -\Delta_{p} u_{2}(z)-\Delta u_{2}(z)+\xi u_{2}(z)^{p-1}=h_{2}(z) \quad \text { in } \Omega .
\end{aligned}
$$

Then $u_{2}-u_{1} \in \operatorname{int} C_{+}$.

Next, for $q \in(1, \infty)$, we recall some basic facts concerning the spectrum of $\left(-\Delta_{q}, W_{0}^{1, q}(\Omega)\right)$. So, we consider the following nonlinear eigenvalue problem

$$
-\Delta_{q} u(z)=\hat{\lambda}|u(z)|^{q-2} u(z) \quad \text { in } \Omega,\left.\quad u\right|_{\partial \Omega}=0 .
$$

A number $\widehat{\lambda} \in \mathbb{R}$ is an eigenvalue of $\left(-\Delta_{q}, W_{0}^{1, q}(\Omega)\right)$ if the above equation admits a nontrivial solution $\widehat{u} \in W_{0}^{1, q}(\Omega)$, which is an eigenfunction corresponding to the eigenvalue $\hat{\lambda}$. We know that $\left(-\Delta_{q}, W_{0}^{1, q}(\Omega)\right)$ admits a smallest eigenvalue $\widehat{\lambda}_{1}(q)$, which has the following properties:

(i) $\widehat{\lambda}_{1}(q)>0$;

(ii) $\widehat{\lambda}_{1}(q)$ is isolated, that is there exists $\varepsilon>0$ such that $\left[\widehat{\lambda}_{1}(q), \widehat{\lambda}_{1}(q)+\varepsilon\right)$ does not contain any other eigenvalue of $\left(-\Delta_{q}, W_{0}^{1, q}(\Omega)\right)$;

(iii) $\hat{\lambda}_{1}(q)$ is simple, that is, if $u, v$ are eigenfunctions corresponding to the eigenvalue $\widehat{\lambda}_{1}(q)>0$, then $u=\xi v$ for some $\xi \neq 0$;

(iv) $\widehat{\lambda}_{1}(q)$ admits the following variational characterization

$$
\widehat{\lambda}_{1}(q)=\inf \left\{\frac{\|D u\|_{q}^{q}}{\|u\|_{q}^{q}}: u \in W_{0}^{1, q}(\Omega), u \neq 0\right\} .
$$

In relation (2.4) the infimum is attained on the one-dimensional eigenspace of $\hat{\lambda}_{1}(q)$. It is clear from (2.4) that the elements of this eigenspace do not change sign. By $\widehat{u}_{1}(q)$ we denote the $L^{q}$-normalized (that is, $\left\|\widehat{u}_{1}(q)\right\|_{q}=1$ ) positive eigenfunction corresponding to $\widehat{\lambda}_{1}(q)$. In fact, $\widehat{\lambda}_{1}(q)>0$ is the only eigenvalue with eigenfunctions of constant sign. All the other eigenvalues have nodal eigenfunctions. The nonlinear regularity theory (see for example, Gasinski 
and Papageorgiou [13, pp. 737-738]) implies that $\widehat{u}_{1}(q) \in C_{+} \backslash\{0\}$. The nonlinear maximum principle of Vazquez [31] implies that $\widehat{u}_{1}(q) \in \operatorname{int} C_{+}$.

Let $\widehat{\sigma}(q)$ denote the spectrum (i.e. the set of eigenvalues) of $\left(-\Delta_{q}, W_{0}^{1, q}(\Omega)\right)$. We can easily check that $\widehat{\sigma}(q)$ is closed. Since $\widehat{\sigma}(q)$ is closed and $\widehat{\lambda}_{1}(q)>0$ is isolated, the second eigenvalue $\widehat{\lambda}_{2}(q)$ of $\left(-\Delta_{q}, W_{0}^{1, q}(\Omega)\right)$ is well defined by

$$
\widehat{\lambda}_{2}(q)=\inf \left\{\widehat{\lambda} \in \widehat{\sigma}(q): \widehat{\lambda}>\widehat{\lambda}_{1}(q)\right\}
$$

The Lusternik-Schnirelmann minimax scheme gives a whole sequence $\left\{\widehat{\lambda}_{k}(q)\right\}_{k \geq 1}$ of distinct eigenvalues such that $\widehat{\lambda}_{k}(q) \rightarrow+\infty$ as $k \rightarrow+\infty$. If $p=2$ or $N=1$ these are all the elements of $\widehat{\sigma}(q)$. Otherwise we do not know if this is the complete list of eigenvalues.

The Lusternik-Schnirelmann scheme provides a minimax characterization of $\widehat{\lambda}_{2}(q)$. For our purposes, this characterization is not convenient. Instead we will use an alternative one due to Cuesta, de Figueiredo and Gossez [8]. So, let

$$
\begin{gathered}
\partial B_{1}^{L^{q}}=\left\{u \in L^{q}(\Omega):\|u\|_{q}=1\right\}, \quad M=W_{0}^{1, q}(\Omega) \cap \partial B_{1}^{L^{q}} \\
\widehat{\Gamma}=\left\{\widehat{\gamma} \in C([-1,1], M): \widehat{\gamma}(-1)=-\widehat{u}_{1}(q), \widehat{\gamma}(1)=\widehat{u}_{1}(q)\right\} .
\end{gathered}
$$

Proposition 2.9. We have $\widehat{\lambda}_{2}(q)=\inf _{\widehat{\lambda} \in \widehat{\Gamma}} \max _{-1 \leq t \leq 1}\|D \widehat{\gamma}(t)\|_{q}^{q}$.

As we already mentioned, in the particular case of $(p, 2)$-equations, using also tools from Morse theory (critical groups), we will be able to generate two nodal solutions, for a total of four nontrivial solutions. So, let us briefly recall some basic relevant definitions and facts from Morse theory.

Let $X$ be a Banach space and $Y_{2} \subseteq Y_{1} \subseteq X$. For any integer $k \geq 0$, we denote by $H_{k}\left(Y_{1}, Y_{2}\right)$ the $k$ th relative singular homology group for the pair $\left(Y_{1}, Y_{2}\right)$ with integer coefficients. Recall that $H_{k}\left(Y_{1}, Y_{2}\right)=0$ for all integers $k<0$.

Given $\varphi \in C^{1}(X)$ and $c \in \mathbb{R}$, we introduce the following sets:

$$
\begin{aligned}
\varphi^{c} & =\{x \in X: \varphi(x) \leq c\}, \\
K_{\varphi} & =\left\{x \in X: \varphi^{\prime}(x)=0\right\}, \quad K_{\varphi}^{c}=\left\{x \in K_{\varphi}: \varphi(x)=c\right\} .
\end{aligned}
$$

The critical groups of $\varphi$ at an isolated critical point $x_{0} \in X$ with $\varphi\left(x_{0}\right)=c$ (that is, $x_{0} \in K_{\varphi}^{c}$ ) are defined by

$$
C_{k}\left(\varphi, x_{0}\right)=H_{k}\left(\varphi^{c} \cap U, \varphi^{c} \cap U \backslash\left\{x_{0}\right\}\right) \quad \text { for all } k \geq 0,
$$

where $U$ is a neighbourhood of $x_{0}$ such that $\varphi^{c} \cap K_{\varphi} \cap U=\left\{x_{0}\right\}$. The excision property of singular homology theory implies that the above definition of critical groups is independent of the particular choice of the neighbourhood $U$.

Suppose that $\varphi \in C^{1}(X)$ satisfies the PS-condition and $\inf \varphi\left(K_{\varphi}\right)>-\infty$. Let $c<\inf \varphi\left(K_{\varphi}\right)$. Then the critical groups of $\varphi$ at infinity are defined by

$$
C_{k}(\varphi, \infty)=H_{k}\left(X, \varphi^{c}\right) \text { for all } k \geq 0 .
$$


The second deformation theorem (see, for example, Gasinski and Papageorgiou [14, p. 628]) implies that the above definition of critical groups at infinity is independent of the choice of the level $c<\inf \varphi\left(K_{\varphi}\right)$.

Assume that $K_{\varphi}$ is finite. We introduce the following polynomials in $t \in \mathbb{R}$ :

$$
M(t, x)=\sum_{k \geq 0} \operatorname{rank} C_{k}(\varphi, x) t^{k}, \quad P(t, \infty)=\sum_{k \geq 0} \operatorname{rank} C_{k}(\varphi, \infty) t^{k} .
$$

Then the Morse relation says that

$$
\sum_{x \in K_{\varphi}} M(t, x)=P(t, \infty)+(1+t) Q(t),
$$

where $Q(t)=\sum_{K \geq 0} \beta_{k} t^{k}$ is a formal series with nonnegative integer coefficients $\beta_{k}$.

For every $x \in \mathbb{R}$, we set $x^{ \pm}=\max \{ \pm x, 0\}$. Then for $u \in W_{0}^{1, p}(\Omega)$ we define $u^{ \pm}(\cdot)=u(\cdot)^{ \pm}$. We know that

$$
u=u^{+}-u^{-}, \quad|u|=u^{+}+u^{-} \quad \text { and } \quad u^{ \pm} \in W_{0}^{1, p}(\Omega) .
$$

Given a measurable function $h: \Omega \times \mathbb{R} \rightarrow \mathbb{R}$ (for example, a Carathéodory function), we define

$$
N_{h}(u)(\cdot)=h(\cdot, u(\cdot)) \quad \text { for all } u \in W_{0}^{1, p}(\Omega) .
$$

Recall that $\|\cdot\|$ denotes the norm of the Sobolev space $W_{0}^{1, p}(\Omega)$, hence $\|u\|=\|D u\|_{p}$ for all $u \in W_{0}^{1, p}(\Omega)$. The same notation will also be used to denote the norm of $\mathbb{R}^{N}$. However, no confusion is possible, since it will always be clear from the context what norm we mean. Finally, we denote by $|\cdot|_{N}$ the Lebesgue measure on $\mathbb{R}^{N}$.

\section{Three Nontrivial Solutions}

In this section we prove the existence of three nontrivial solutions for problem (1.1) and provide sign information for all of them.

We assume that the reaction term $f(z, x)$ is a Carathéodory function $f: \Omega \times$ $\mathbb{R} \rightarrow \mathbb{R}$ such that $f(z, 0)=0$ for almost all $z \in \Omega$ and the following hypotheses are fulfilled:

$\left(\mathrm{H}_{1}\right) \quad$ (i) for every $\rho>0$, there exists $a_{\rho} \in L^{\infty}(\Omega)_{+}$such that $|f(z, x)| \leq$ $a_{\rho}(z)$ for almost all $z \in \Omega$ and all $|x| \leq \rho$;

(ii) $\lim _{x \rightarrow \pm \infty} f(z, x) /|x|^{p-2} x=-\infty$ uniformly for almost all $z \in \Omega$;

(iii) if $\tau \in(1, p]$ and $\widetilde{c}>0$ are as in hypothesis $\mathrm{H}(\mathrm{a})(\mathrm{iv})$, then there exist $\delta_{0}>0$ and $c_{6}>\widetilde{c} \widehat{\lambda}_{1}(\tau)$ such that $f(z, x) x \geq c_{6}|x|^{\tau}$ for almost all $z \in \Omega$ and all $|x| \leq \delta_{0}$.

REMARK 3.1. Note that the growth restriction on $f(z, \cdot)\left(\right.$ see $\left.\left(\mathrm{H}_{1}\right)(\mathrm{i})\right)$ is only local. In fact, the only growth restriction on $f(z, \cdot)$ is hypothesis $\left(\mathrm{H}_{1}\right)(\mathrm{ii})$ and the growth to $-\infty$ as $x \rightarrow+\infty$ and to $+\infty$ as $x \rightarrow-\infty$ can be arbitrary. 
When $\tau<p$, hypothesis $\left(\mathrm{H}_{1}\right)$ (iii) reveals the presence of a concave term near zero.

ExAmple 3.2. The following function satisfies hypotheses $\left(\mathrm{H}_{1}\right)$ (for the sake of simplicity, we drop the $z$-dependence):

$$
f(x)=|x|^{\tau-2} x-|x|^{r-2} x \quad \text { with } 1<\tau \leq p<r<\infty .
$$

First, we produce two nontrivial constant sign smooth solutions. To this end, note that we can find $M_{1}>\delta_{0}$ (see hypothesis $\left(\mathrm{H}_{1}\right)(\mathrm{iii})$ ) such that

$$
f(z, x) x \leq-1<0 \quad \text { for a.a. } z \in \Omega \text { and all }|x| \geq M_{1} .
$$

Let $\xi>M_{1}$. Then

$$
\begin{array}{cl}
0=A(\xi) \geq N_{f}(\xi) & \text { in } W^{-1, p^{\prime}}(\Omega), \\
0=A(-\xi) \leq N_{f}(-\xi) & \text { in } W^{-1, p^{\prime}}(\Omega) .
\end{array}
$$

We define the following order intervals in the Sobolev space $W_{0}^{1, p}(\Omega)$ :

$$
\begin{gathered}
{[0, \xi]=\left\{u \in W_{0}^{1, p}(\Omega): 0 \leq u(z) \leq \xi \text { for a.a. } z \in \Omega\right\},} \\
{[-\xi, 0]=\left\{u \in W_{0}^{1, p}(\Omega):-\xi \leq u(z) \leq 0 \text { for a.a. } z \in \Omega\right\} .}
\end{gathered}
$$

We introduce the following truncations of the reaction $f(z, \cdot)$ :

$$
\begin{aligned}
& k_{+}(z, x)= \begin{cases}0 & \text { if } x<0, \\
f(z, x) & \text { if } 0 \leq x \leq \xi, \\
f(z, \xi) & \text { if } \xi<x,\end{cases} \\
& k_{-}(z, x)= \begin{cases}f(z,-\xi) & \text { if } x<-\xi, \\
f(z, x) & \text { if }-\xi \leq x \leq 0, \\
0 & \text { if } 0<x .\end{cases}
\end{aligned}
$$

Both $k_{+}$and $k_{-}$are Carathéodory functions. We set

$$
K_{ \pm}(z, x)=\int_{0}^{x} k_{ \pm}(z, s) d s
$$

and consider the $C^{1}$-functions $\psi_{ \pm}: W_{0}^{1, p}(\Omega) \rightarrow \mathbb{R}$ defined by

$$
\psi_{ \pm}(u)=\int_{\Omega} G(D u(z)) d z-\int_{\Omega} K_{ \pm}(z, u(z)) d z \quad \text { for all } u \in W_{0}^{1, p}(\Omega) .
$$

Let $\varphi: W_{0}^{1, p}(\Omega) \rightarrow \mathbb{R}$ be the energy functional for problem (1.1) defined by

$$
\varphi(u)=\int_{\Omega} G(D u(z)) d z-\int_{\Omega} F(z, u(z)) d z \quad \text { for all } u \in W_{0}^{1, p}(\Omega),
$$

where $F(z, x)=\int_{0}^{x} f(z, s) d s$. 
Proposition 3.3. Assume that hypotheses $\mathrm{H}(\mathrm{a})$ and $\left(\mathrm{H}_{1}\right)$ hold. Then problem (1.1) has at least two nontrivial solutions of constant sign $u_{0} \in[0, \xi] \cap \operatorname{int} C_{+}$ and $v_{0} \in[-\xi, 0] \cap\left(-\operatorname{int} C_{+}\right)$.

Proof. First we produce the nontrivial positive solution. To this end, we consider the functional $\psi_{+}$. It is clear from (3.1)-(3.2) that $\psi_{+}$is coercive. Also, using the Sobolev embedding theorem, we see that $\psi_{+}$is sequentially weakly lower semi-continuous. So, by the Weierstrass theorem, we can find $u_{0} \in W_{0}^{1, p}(\Omega)$ such that

$$
\psi_{+}\left(u_{0}\right)=\inf \left[\psi_{+}(u): u \in W_{0}^{1, p}(\Omega)\right] .
$$

Fix $\varepsilon>0$. By virtue of hypothesis $\mathrm{H}(\mathrm{a})(\mathrm{iv})$, there exists $\delta=\delta(\varepsilon) \leq \delta_{0}$ (see hypothesis $\left.\left(\mathrm{H}_{1}\right)(\mathrm{iii})\right)$ such that

$$
G_{0}(t) \leq \frac{\widetilde{c}+\varepsilon}{\tau} t^{\tau} \quad \text { for all } t \in[0, \delta]
$$

Therefore

$$
G(y) \leq \frac{\widetilde{c}+\varepsilon}{\tau}\|y\|^{\tau} \quad \text { for all }\|y\| \leq \delta, y \in \mathbb{R}^{N} .
$$

Let $t \in(0,1)$ be small such that $t \widehat{u_{1}}(\tau)(z), t|| D \widehat{u_{1}}(\tau)(z) \| \leq \delta$ for all $z \in \bar{\Omega}$ (recall that $\widehat{u}_{1}(\tau) \in \operatorname{int} C_{+}$). Then

$$
\begin{aligned}
\psi_{+}\left(t \widehat{u}_{1}(\tau)\right) & =\int_{\Omega} G\left(t D \widehat{u_{1}}(\tau)\right) d z-\int_{\Omega} K_{+}\left(z, t \widehat{u}_{1}(\tau)\right) d z \\
& \leq \frac{\widetilde{c}+\varepsilon}{\tau} t^{\tau}\left\|D \widehat{u}_{1}\right\|_{\tau}^{\tau}-\int_{\Omega} F\left(z, t \widehat{u}_{1}(\tau)\right) d z
\end{aligned}
$$

(see (3.4) and recall that $\left.\delta \leq \delta_{0}<\xi\right)$

$$
\leq \frac{\widetilde{c}+\varepsilon}{\tau} t^{\tau} \widehat{\lambda}_{1}(\tau)-\frac{c_{6}}{\tau} t^{\tau}
$$

(see $(2.4)$, hypothesis $\left(\mathrm{H}_{1}\right)(\mathrm{iii})$ and recall that $\left\|\widehat{u}_{1}(\tau)\right\|_{\tau}=1$ )

$$
=\frac{t^{\tau}}{\tau}\left[(\widetilde{c}+\varepsilon) \widehat{\lambda}_{1}(\tau)-c_{6}\right] .
$$

Since $c_{6}>\widehat{c}_{1}(\tau)$ (see hypothesis $\left(\mathrm{H}_{1}\right)(\mathrm{iii})$ ), by choosing $\varepsilon \in(0,1)$ small, from (3.5) we infer that

$$
\psi_{+}\left(t \widehat{u}_{1}(\tau)\right)<0 \Rightarrow \psi_{+}\left(u_{0}\right)<0=\psi_{+}(0)
$$

(see $(3.3))$, hence $u_{0} \neq 0$. Relation (3.3) yields

$$
\psi_{+}^{\prime}\left(u_{0}\right)=0 \Rightarrow A\left(u_{0}\right)=N_{k_{+}}\left(u_{0}\right) .
$$

On (3.6) first we act with $-u_{0}^{-} \in W_{0}^{1, p}(\Omega)$ and obtain

$$
\frac{c_{1}}{p-1}\left\|D u_{0}^{-}\right\|_{p}^{p} \leq 0
$$


(see Lemma 2.3(c) and (3.1)-(3.2)), hence $u_{0} \geq 0, u_{0} \neq 0$.

Next, we act on (3.6) with $\left(u_{0}-\xi\right)^{+} \in W_{0}^{1, p}(\Omega)$. Thus, by (3.1)-(3.2) and (3.2),

$$
\left\langle A\left(u_{0}\right),\left(u_{0}-\xi\right)^{+}\right\rangle=\int_{\Omega} k_{+}\left(z, u_{0}\right)\left(u_{0}-\xi\right)^{+} d z=\int_{\Omega} f(z, \xi)\left(u_{0}-\xi\right)^{+} d z \leq 0 .
$$

It follows that

$$
\int_{\left\{u_{0}>\xi\right\}}\left(a\left(D u_{0}\right), D u_{0}\right)_{\mathbb{R}^{N}} d z \leq 0 \Rightarrow\left|\left\{u_{0}>\xi\right\}\right|_{N}=0,
$$

hence $u_{0} \leq \xi$. So, we have proved that $u_{0} \in[0, \xi]$. Relation (3.6) becomes

$$
A\left(u_{0}\right)=N_{f}\left(u_{0}\right) \Rightarrow-\operatorname{div} a\left(D u_{0}(z)\right)=f\left(z, u_{0}(z)\right) \quad \text { a.e. in } \Omega,\left.\quad u_{0}\right|_{\partial \Omega}=0
$$

(see (3.1)-(3.2)).

From Ladyzhenskaya and Ural'tseva [17, p. 286], we have $u_{0} \in L^{\infty}(\Omega)$. Then, using the regularity results of Lieberman [18, p. 320], we deduce that $u_{0} \in$ $C_{+} \backslash\{0\}$. Since $u_{0} \in[0, \xi]$, using hypotheses $\left(\mathrm{H}_{1}\right)(\mathrm{i})$, (iii), for $r \in\left(p, p^{*}\right)$ we can find $c_{7}=c_{7}(r, \xi)>0$ such that

$$
f(z, x) x \geq c_{6}|x|^{\tau}-c_{7}|x|^{r} \quad \text { for a.a. } z \in \Omega \text { and all }|x| \leq \xi .
$$

Therefore from (3.7) and since $u_{0} \in C_{+} \backslash\{0\}$, we have

$$
-\operatorname{div} a\left(D u_{0}(z)\right)+c_{7} u_{0}(z)^{r-1}=f\left(z, u_{0}(z)\right)+c_{6} u_{0}(z)^{\tau-1} \geq c_{6} u_{0}(z)^{\tau-1} \geq 0
$$

almost everywhere in $\Omega$. It follows that

$$
\operatorname{div} a\left(D u_{0}(z)\right) \leq c_{7}\left\|u_{0}\right\|_{\infty}^{r-p} u_{0}(z)^{p-1} \quad \text { a.e. in } \Omega .
$$

The nonlinear strong maximum principle of Pucci and Serrin [27, p. 111] implies that $u_{0}(z)>0$ for all $z \in \Omega$. Thus, by the boundary point theorem of Pucci and Serrin $\left[27\right.$, p. 120] we conclude that $u_{0} \in \operatorname{int} C_{+}$. Similarly, working this time with the functional $\psi_{-}$, we produce a second nontrivial constant sign solution $v_{0} \in[-\xi, 0] \cap\left(-\operatorname{int} C_{+}\right)$.

If we strengthen a little the conditions on the reaction term $f(z, \cdot)$, we can say more about the two constant sign smooth solutions produced in Proposition 3.3. The new stronger hypotheses on the nonlinearity $f(z, x)$ are the following:

$\left(\mathrm{H}_{2}\right) \quad f: \Omega \times \mathbb{R} \rightarrow \mathbb{R}$ is a Carathéodory function such that $f(z, 0)=0$ for almost all $z \in \Omega$, hypotheses $\left(\mathrm{H}_{2}\right)(\mathrm{i})$-(iii) are the same as the corresponding hypotheses $\left(\mathrm{H}_{1}\right)(\mathrm{i})-(\mathrm{iii})$, and

(iv) for every $\rho>0$, there exists $\xi_{\rho}>0$ such that for almost all $z \in \Omega$ the function $x \mapsto f(z, x)+\xi_{\rho}|x|^{p-2} x$ is nondecreasing on $[-\rho, \rho]$. 
Proposition 3.4. Assume that hypotheses $\mathrm{H}(\mathrm{a})$ and $\left(\mathrm{H}_{2}\right)$ hold. Then problem (1.1) has at least two nontrivial solutions of constant sign $u_{0} \in[0, \xi] \cap \operatorname{int} C_{+}$ and $v_{0} \in[-\xi, 0] \cap\left(\right.$ int $\left.C_{+}\right)$. Moreover, both $u_{0}$ and $v_{0}$ are local minimizers of the energy functional $\varphi$.

Proof. By virtue of Proposition 3.3, we already have two nontrivial constant sign solutions $u_{0} \in[0, \xi] \cap \operatorname{int} C_{+}$and $v_{0} \in[-\xi, 0] \cap\left(-\operatorname{int} C_{+}\right)$.

Hypothesis $\left(\mathrm{H}_{2}\right)(\mathrm{iv})$ implies that we can find $\widehat{\xi}>0$ such that for almost all $z \in \Omega$, the function $x \mapsto f(z, x)+\widehat{\xi}|x|^{p-2} x$ is nondecreasing on $[-\xi, \xi]$.

Let $\delta>0$ and set $u_{\delta}(z)=u_{0}(z)+\delta$ for all $z \in \bar{\Omega}$. Then $u_{\delta} \in C^{1}(\bar{\Omega})$ and

$$
-\operatorname{div} a\left(D u_{\delta}(z)\right)+\widehat{\xi} u_{\delta}(z)^{p-1} \leq-\operatorname{div} a\left(D u_{0}(z)\right)+\widehat{\xi} u_{0}(z)^{p-1}+\gamma(\delta)
$$

with $\gamma(\delta) \rightarrow 0^{+}$as $\delta \rightarrow 0^{+}$

$$
=f\left(z, u_{0}(z)\right)+\widehat{\xi} u_{0}(z)^{p-1}+\gamma(\delta) \leq f(z, \xi)+\widehat{\xi} \xi^{p-1}+\gamma(\delta)
$$

(since $\left.u_{0} \in[0, \xi]\right)$

$$
\leq \widehat{\xi}^{p-1}
$$

for $\delta>0$ small (recall that $f(z, \xi) \leq-1 / \xi$ for almost all $z \in \Omega$ )

$$
=-\operatorname{div} a(D \xi)+\widehat{\xi} \xi^{p^{-1}}
$$

almost everywhere in $\Omega$.

Thus, $u_{\delta}(z) \leq \xi$ for all $z \in \bar{\Omega}$ (by the the weak comparison principle). Hence we conclude that $u_{0}(z)<\xi$ for all $z \in \bar{\Omega}$. Since $u_{0} \in \operatorname{int} C_{+}$, it follows that

$$
u_{0} \in \operatorname{int}_{C_{0}^{1}(\bar{\Omega})}[0, \xi] .
$$

Notice that $\left.\psi_{+}\right|_{[0, \xi]}=\left.\varphi\right|_{[0, \xi]}$. So, it follows that $u_{0}$ is a local $C_{0}^{1}(\bar{\Omega})$-minimizer of $\varphi$. Invoking Proposition 2.7 it follows that $u_{0}$ is a local $W_{0}^{1, p}(\Omega)$-minimizer of $\varphi_{-}$. Similarly, for $v_{0} \in[-\xi, 0] \cap\left(-\operatorname{int} C_{+}\right)$, using this time the functional $\psi$.

In fact, we can produce extremal nontrivial constant sign solutions for problem (1.1), that is, the smallest nontrivial positive solution and the biggest nontrivial negative solution. We follow the reasoning on Papageorgiou and Rădulescu [25].

From the proof of Proposition 3.3, we know that

$$
f(z, x) x \geq c_{6}|x|^{\tau}-c_{7}|x|^{r} \quad \text { for a.a. } z \in \Omega \text { and all }|x| \leq \xi .
$$

This growth estimate leads to the following auxiliary Dirichlet problem:

$$
-\operatorname{div} a(D u(z))=c_{6}|u(z)|^{\tau-2} u(z)-c_{7}|u(z)|^{r-2} u(z) \quad \text { in } \Omega,\left.\quad u\right|_{\partial \Omega}=0 .
$$


Proposition 3.5. Assume that hypotheses $\mathrm{H}(\mathrm{a})$ hold. Then problem (3.9) has a unique nontrivial positive solution $\widetilde{u} \in \operatorname{int} C_{+}$and since problem (3.9) is odd, $\widetilde{v}=-\widetilde{u} \in-\operatorname{int} C_{+}$is the unique nontrivial negative solution of (3.9).

Proof. First we establish the existence of a nontrivial positive solution for problem (3.9). To this end, let $\sigma_{+}: W_{0}^{1, p}(\Omega) \rightarrow \mathbb{R}$ be the $C^{1}$-functional defined by

$$
\sigma_{+}(u)=\int_{\Omega} G(D u(z)) d z-\frac{c_{6}}{\tau}\left\|u^{+}\right\|_{\tau}^{\tau}+\frac{c_{7}}{r}\left\|u^{+}\right\|^{r} \quad \text { for all } u \in W_{0}^{1, p}(\Omega) .
$$

Since $r>p \geq \tau$, from Corollary 2.4 we deduce that $\sigma_{+}(\cdot)$ is coercive and sequentially weakly lower semi-continuous. Thus, we can find $\widetilde{u} \in W_{0}^{1, p}(\Omega)$ such that

$$
\sigma_{+}(\widetilde{u})=\inf \left\{\sigma_{+}(u): u \in W_{0}^{1, p}(\Omega)\right\}
$$

As before (see the proof of Proposition 3.3), we choose $t \in(0,1)$ small such that

$$
t \widehat{u}_{1}(\tau)(z), t|| D \widehat{u}_{1}(\tau)(z) \| \leq \delta \quad \text { for all } z \in \bar{\Omega}
$$

with $\delta \in\left(0, \delta_{0}\right]$ as in $(3.4)$. Then we have

$$
\begin{aligned}
\sigma_{+}\left(t \widehat{u}_{1}(\tau)\right) & \leq \frac{\widetilde{c}+\varepsilon}{\tau} t^{\tau} \widehat{\lambda}_{1}(\tau)-\frac{c_{6}}{\tau} t^{\tau}+\frac{c_{7}}{r} t^{r}\left\|\widehat{u}_{1}(\tau)\right\|_{r}^{r} \\
& =\frac{t^{\tau}}{\tau}\left[(\widetilde{c}+\varepsilon) \widehat{\lambda}_{1}(\tau)-c_{6}\right]+\frac{c_{7}}{r} t^{r}\left\|\widehat{u}_{1}(\tau)\right\|_{r}^{r} .
\end{aligned}
$$

Since $c_{6}>\widehat{c}_{\lambda_{1}}(\tau)$, by choosing $\varepsilon \in(0,1)$ small and since $r>\tau$, by choosing $t \in(0,1)$ even smaller if necessary, from $(3.11)$ we see that $\sigma_{+}\left(t \widehat{u}_{1}(\tau)\right)<0$. Taking into account (3.10) and since $\sigma_{+}(0)=0$, we deduce that $\widetilde{u} \neq 0$.

From (3.11) we have

$$
\sigma_{+}^{\prime}(\widetilde{u})=0 \Rightarrow A(\widetilde{u})=c_{6}\left(\widetilde{u}^{+}\right)^{\tau-1}-c_{7}\left(\widetilde{u}^{+}\right)^{r-1} .
$$

On (3.12) we act with $-\widetilde{u}^{-} \in W_{0}^{1, p}(\Omega)$. Then using Lemma 2.3(c), we have

$$
\frac{c_{1}}{p-1}\left\|D \widetilde{u}^{-}\right\|_{p}^{p} \leq 0
$$

hence $\widetilde{u} \geq 0, \widetilde{u} \neq 0$. Then relation (3.12) becomes

$$
\begin{aligned}
A(\widetilde{u})=c_{6} & \widetilde{u}^{\tau-1}-c_{7} \widetilde{u}^{r-1} \\
& \Rightarrow-\operatorname{div} a(D \widetilde{u}(z))=c_{6} \widetilde{u}(z)^{\tau-1}-c_{7} \widetilde{u}(z)^{r-1} \text { a.e. in } \Omega,\left.\quad \widetilde{u}\right|_{\partial \Omega}=0 .
\end{aligned}
$$

As before, the nonlinear regularity theory (see [17], [18]), implies that $\widetilde{u} \in$ $C_{+} \backslash\{0\}$. We have

$$
\operatorname{div} a(D \widetilde{u}(z)) \leq c_{7}\|\widetilde{u}\|_{\infty}^{r-p} \widetilde{u}(z)^{p-1} \text { a.e. in } \Omega \Rightarrow \widetilde{u} \in \operatorname{int} C_{+}
$$

(see Pucci and Serrin [27, pp. 111, 120]). 
Now we show the uniqueness of this positive solution. To this end, we introduce the integral functional $\gamma_{+}: L^{1}(\Omega) \rightarrow \overline{\mathbb{R}}=\mathbb{R} \cup\{+\infty\}$ defined by

$$
\gamma_{+}(u)= \begin{cases}\int_{\Omega} G\left(D u^{1 / \tau}\right) d t & \text { if } u \geq 0, u^{1 / \tau} \in W_{0}^{1, p}(\Omega), \\ +\infty & \text { otherwise. }\end{cases}
$$

Let $u_{1}, u_{2} \in \operatorname{dom} \gamma_{+}$and set $v_{1}=u_{1}^{1 / \tau}, v_{2}=u_{2}^{1 / \tau}$. We have $v_{1}, v_{2} \in W_{0}^{1, p}(\Omega)$, see (3.13). We set

$$
v=\left(t u_{1}+(1-t) u_{2}\right)^{1 / \tau} \quad \text { with } t \in[0,1] .
$$

Invoking Lemma 1 of Diaz and Saa [9], we have

$$
\|D v(z)\| \leq\left(t\left\|D v_{1}(z)\right\|^{\tau}+(1-t)\left\|D v_{2}(z)\right\|^{\tau}\right)^{1 / \tau} \quad \text { for a.a. } z \in \Omega .
$$

Since $G_{0}$ is increasing, we have

$$
G_{0}(\|D v(z)\|) \leq G_{0}\left(\left(t\left\|D v_{1}(z)\right\|^{\tau}+(1-t)\left\|D v_{2}(z)\right\|^{\tau}\right)^{1 / \tau}\right) \quad \text { for a.a. } z \in \Omega .
$$

From hypothesis $\mathrm{H}(\mathrm{a})(\mathrm{iv})$, we know that $t \mapsto G_{0}\left(t^{1 / \tau}\right)$ is convex in $(0,+\infty)$. It follows that

$$
\begin{array}{ll}
G_{0}\left(\left(t\left\|D v_{1}(z)\right\|^{\tau}+(1-t)\left\|D v_{2}(z)\right\|^{\tau}\right)^{1 / \tau}\right) & \\
\leq t G_{0}\left(\| D v_{1}(z)\right) \|+(1-t) G_{0}\left(\left\|D v_{2}(z)\right\|\right) & \text { a.e. in } \Omega \\
\Rightarrow G(D v(z)) \leq t G\left(D v_{1}(z)\right)+(1-t) G\left(D v_{2}(z)\right) & \text { a.e. in } \Omega \\
\Rightarrow \gamma_{+} \text {is convex. } &
\end{array}
$$

Also, by Fatou's lemma, we see that $\gamma_{+}$is lower semi-continuous.

Let $u \in W_{0}^{1, p}(\Omega)$ be a nontrivial positive solution of problem (3.9). From the first part of the proof we know that $u$ exists and $u \in \operatorname{int} C_{+}$. We have $u^{\tau} \geq 0,\left(u^{\tau}\right)^{1 / \tau}=u \in W_{0}^{1, p}(\Omega)$. Hence $u^{\tau} \in \operatorname{dom} \gamma_{+}$. Let $h \in C_{0}^{1}(\bar{\Omega})$. Then for $t \in(-1,1)$ with $|t|$ small, we have $u^{\tau}+t h \in \operatorname{int} C_{+}$and so $u^{\tau}+t h \in \operatorname{dom} \gamma_{+}$. Therefore, the Gâteaux derivative of $\gamma_{+}$at $u^{\tau}$ in the direction $h$ exists and by the chain rule,

$$
\gamma_{+}^{\prime}\left(u^{\tau}\right)(h)=\frac{1}{\tau} \int_{\Omega} \frac{-\operatorname{div} a(D u)}{u^{\tau-1}} h d z .
$$

If $v \in W_{0}^{1, p}(\Omega)$ is another nontrivial positive solution of (3.9), then as above we have $v \in \operatorname{int} C_{+}$and

$$
\gamma_{+}^{\prime}\left(v^{\tau}\right)(h)=\frac{1}{\tau} \int_{\Omega} \frac{-\operatorname{div} a(D v)}{v^{\tau-1}} h d z .
$$

The convexity of $\gamma_{+}$implies the monotonicity of $\gamma_{+}^{\prime}$. Hence

$$
\begin{aligned}
0 & \leq \frac{1}{\tau} \int_{\Omega}\left(\frac{-\operatorname{div} a(D u)}{u^{\tau-1}}+\frac{\operatorname{div} a(D v)}{v^{\tau-1}}\right)\left(u^{\tau}-v^{\tau}\right) d z \\
& =\frac{c_{7}}{\tau} \int_{\Omega}\left(v^{r-\tau}-u^{r-\tau}\right)\left(u^{\tau}-v^{\tau}\right) d z \leq 0
\end{aligned}
$$


hence $u=v$. It follows that $\widetilde{u} \in \operatorname{int} C_{+}$is the unique nontrivial positive solution of (3.9). Since (3.9) is odd, $\widetilde{v}=-\widetilde{u} \in-\operatorname{int} C_{+}$is the unique nontrivial negative solution of (3.9).

Using the solutions produced in Proposition 3.5, we can generate extremal constant sign solutions of problem (1.1).

Proposition 3.6. Assume that hypotheses $\mathrm{H}(\mathrm{a})$ and $\left(\mathrm{H}_{1}\right)$ hold. Then problem (1.1) has a smallest nontrivial positive solution $u_{*} \in \operatorname{int} C_{+}$and a biggest nontrivial negative solution $v_{*} \in-\operatorname{int} C_{+}$.

Proof. First we produce $u_{*} \in \operatorname{int} C_{+}$, the smallest nontrivial positive solution of (1.1). Let $S_{+}$be the set of nontrivial positive solutions of (1.1) in the order interval $[0, \xi]$. From Proposition 3.3 and its proof we have $S_{+} \neq \varnothing$ and $S_{+} \subseteq[0, \xi] \cap \operatorname{int} C_{+}$. Moreover, as in Filippakis, Kristaly \& Papageorgiou [11], we have that $S_{+}$is downward directed, that is, if $u_{1}, u_{2} \in S_{+}$, then we can find $u \in S_{+}$such that $u \leq u_{1}, u \leq u_{2}$.

Claim 1. $\widetilde{u} \leq u$ for all $u \in S_{+}$.

Let $u \in S_{+}$and let

$$
\eta_{+}(z, x)= \begin{cases}0 & \text { if } x<0 \\ c_{6} x^{\tau-1}-c_{7} x^{r-1} & \text { if } 0 \leq x \leq u(z), \\ c_{6}(z)^{\tau-1}-c_{7} u(z)^{r-1} & \text { if } u(z)<x\end{cases}
$$

Then $\eta_{+}$is a Carathéodory function. We set $H_{+}(z, x)=\int_{0}^{x} \eta_{+}(z, s) d s$ and consider the $C^{1}$-functional $\beta_{+}: W_{0}^{1, p}(\Omega) \rightarrow \mathbb{R}$ defined by

$$
\beta_{+}(y)=\int_{\Omega} G(D y(z)) d z-\int_{\Omega} H_{+}(z, y(z)) d z \quad \text { for all } y \in W_{0}^{1, p}(\Omega) .
$$

It is clear from (3.16) and Corollary 2.4 that $\beta_{+}$is coercive. Also, it is sequentially weakly lower semi-continuous. So, we can find $\widetilde{u}_{0} \in W_{0}^{1, p}(\Omega)$ such that

$$
\beta_{+}\left(\widetilde{u}_{0}\right)=\inf \left\{\beta_{+}(u): u \in W_{0}^{1, p}(\Omega)\right\} .
$$

As before, via hypothesis $\mathrm{H}(\mathrm{a})(\mathrm{iv})$, we show that $\beta_{+}\left(\widetilde{u}_{0}\right)<0=\beta_{+}(0)$, hence $\widetilde{u}_{0} \neq 0$.

From (3.17) we have

$$
\beta_{+}^{\prime}\left(\widetilde{u}_{0}\right)=0 \Rightarrow A\left(\widetilde{u}_{0}\right)=N_{\eta_{+}}\left(\widetilde{u}_{0}\right) .
$$

Acting on (3.18) with $-\widetilde{u}_{0}^{-},\left(\widetilde{u}_{0}-u\right)^{+} \in W_{0}^{1, p}(\Omega)$, we show that

$$
\widetilde{u}_{0} \in[0, u]=\left\{y \in W_{0}^{1, p}(\Omega): 0 \leq y(z) \leq u(z) \text { a.e. in } \Omega\right\} .
$$


Therefore (3.18) becomes

$$
\begin{aligned}
A\left(\widetilde{u}_{0}\right)=c_{6} \widetilde{u}_{0}^{\tau-1}-c_{7} \widetilde{u}_{0}^{r-1} & \Rightarrow \widetilde{u}_{0} \text { is a positive solution of }(3.9) \\
& \Rightarrow \widetilde{u}_{0}=\widetilde{u} \quad(\text { see Proposition } 3.5) \\
& \Rightarrow \widetilde{u} \leq u \text { for all } u \in S_{+} .
\end{aligned}
$$

This proves Claim 1.

Let $C \subseteq S_{+}$be a chain (that is, a totally ordered subset of $S_{+}$). From Dunford \& Schwartz [10, p. 336], we know we can find $\left\{u_{n}\right\}_{n \geq 1} \subseteq C$ such that

$$
\inf C=\inf _{n \geq 1} u_{n}
$$

We have

$$
A\left(u_{n}\right)=N_{f}\left(u_{n}\right), \quad \widetilde{u} \leq u_{n} \leq \xi \quad \text { for all } n \geq 1
$$

(see the Claim 1). Evidently, $\left\{u_{n}\right\}_{n \geq 1} \subseteq W_{0}^{1, p}(\Omega)$ is bounded. So, we may assume that

$$
u_{n} \stackrel{w}{\longrightarrow} u \quad \text { in } W_{0}^{1, p}(\Omega) \quad \text { and } \quad u_{n} \rightarrow u \quad \text { in } L^{\rho}(\Omega) .
$$

We act with $u_{n}-u \in W_{0}^{1, p}(\Omega)$, pass to the limit as $n \rightarrow \infty$, and use (3.20). We obtain

$$
\lim _{n \rightarrow \infty}\left\langle A\left(u_{n}\right), u_{n}-u\right\rangle=0 \Rightarrow u_{n} \rightarrow u \quad \text { in } W_{0}^{1, p}(\Omega)
$$

(see Proposition 2.6). So, if we pass to the limit in (3.19) as $n \rightarrow \infty$ and use (3.21), we deduce that

$$
A(u)=N_{f}(u), \widetilde{u} \leq u \leq \xi \Rightarrow u \in S_{+} \text {and } u=\inf C .
$$

Since $C$ is an arbitrary chain, from the Kuratowski-Zorn lemma we can find $u_{*} \in S_{+} \subseteq$ int $C_{+}$a minimal element. The fact that $S_{+}$is downward directed implies that $u_{*} \in \operatorname{int} C_{+}$is the smallest nontrivial positive solution of problem (1.1).

Similarly, let $S_{-}$be the set of nontrivial negative solutions of $(1.1)$ in $[-\xi, 0]$. We have that $S_{-} \neq \emptyset$ and $S_{-} \subseteq-\operatorname{int} C_{+}$. Also, $S_{-}$is upward directed (that is, if $v_{1}, v_{2} \in S_{-}$then there exists $v \in S_{-}$such that $\left.v_{1} \leq v, v_{2} \leq v\right)$. The previous argument based on the Kuratowski-Zorn lemma leads to the biggest nontrivial negative solution $v_{*} \in-\operatorname{int} C_{+}$of problem (1.1).

The extremal nontrivial constant sign solutions of (1.1) lead to the existence of a nodal solution. For this purpose we need to slightly strengthen hypotheses $\left(\mathrm{H}_{1}\right)$. The new conditions on $f(z, x)$ are the following:

$\left(\mathrm{H}_{1}^{\prime}\right) f: \Omega \times \mathbb{R} \rightarrow \mathbb{R}$ is a Carathéodory function such that $f(z, 0)=0$ for almost all $z \in \Omega$, hypotheses $\left(\mathrm{H}_{1}^{\prime}\right)(\mathrm{i})$, (ii) are the same as the corresponding hypotheses $\left(\mathrm{H}_{1}\right)(\mathrm{i})$, (ii) and 
(iii) if $\tau \in(1, p]$ and $\widetilde{c}>0$ are as in hypothesis $\mathrm{H}($ a)(iv), then there are $\delta_{0}>0$ and $c_{6}>\widetilde{c} \widehat{\lambda}_{2}(\tau)$ such that

$$
f(z, x) x \geq c_{6}|x|^{\tau} \quad \text { for a.a. } z \in \Omega \text { and all }|x| \leq \delta_{0} .
$$

Proposition 3.7. Assume that hypotheses $\mathrm{H}(\mathrm{a})$ and $\left(\mathrm{H}_{1}^{\prime}\right)$ hold. Then problem (1.1) admits a nodal solution $y_{0} \in\left[v_{*}, u_{*}\right] \cap C_{0}^{1}(\bar{\Omega})$.

Proof. Let $u_{*} \in \operatorname{int} C_{+}$and $v_{*} \in-\operatorname{int} C_{+}$be the two extremal constant sign solutions of (1.1) produced in Proposition 3.6. We introduce the following truncation of $f(z, \cdot)$ :

$$
h(z, x)= \begin{cases}f\left(z, v_{*}(z)\right) & \text { if } x<v_{*}(z), \\ f(z, x) & \text { if } v_{*}(z) \leq x \leq u_{*}(z), \\ f\left(z, u_{*}(z)\right) & \text { if } u_{*}(z)<x .\end{cases}
$$

Set $h_{ \pm}(z, x)=h\left(z, \pm x^{ \pm}\right)$. Both are Carathéodory functions. We also define

$$
H(z, x)=\int_{0}^{x} h(z, s) d s \quad \text { and } \quad H_{ \pm}(z, x)=\int_{0}^{x} h_{ \pm}(z, s) d s
$$

and introduce the $C^{1}$-functionals $\widehat{\varphi}, \widehat{\varphi}_{ \pm}: W_{0}^{1, p}(\Omega) \rightarrow \mathbb{R}$ defined by

$$
\begin{aligned}
\widehat{\varphi}(u) & =\int_{\Omega} G(D u(z)) d z-\int_{\Omega} H(z, u(z)) d z \\
\widehat{\varphi_{ \pm}}(u) & =\int_{\Omega} G(D u(z)) d z-\int_{\Omega} H_{ \pm}(z, u(z)) d z \quad \text { for all } u \in W_{0}^{1, p}(\Omega) .
\end{aligned}
$$

We can easily check as before that

$$
K_{\widehat{\varphi}} \subseteq\left[v_{*}, u_{*}\right], \quad K_{\widehat{\varphi}_{+}}=\left\{0, u_{*}\right\}, \quad K_{\widehat{\varphi}_{-}}=\left\{v_{*}, 0\right\}
$$

(recall the extremality of $u_{*} \in \operatorname{int} C_{+}$and $v_{*} \in-\operatorname{int} C_{+}$). Note that $\hat{\varphi}_{+}$is coercive (see (3.22)) and sequentially weakly lower semi-continuous. So, there exists $\widehat{u}_{*} \in W_{0}^{1, p}(\Omega)$ such that

$$
\begin{array}{r}
\widehat{\varphi}_{+}\left(\widehat{u}_{*}\right)=\inf \left\{\widehat{\varphi}_{+}(u): u \in W_{0}^{1, p}(\Omega)\right\}<0=\widehat{\varphi}_{+}(0) \quad(\text { as before, see H(a)(iv)) } \\
\Rightarrow \widehat{u}_{*} \in K_{\widehat{\varphi}_{+}} \backslash\{0\},
\end{array}
$$

hence $\widehat{u}_{*}=u_{*} \in \operatorname{int} C_{+}$, see (3.23).

Since $\left.\hat{\varphi}\right|_{C_{+}}=\left.\widehat{\varphi}_{+}\right|_{C_{+}}$, it follows that $u_{*}$ is a local $C_{0}^{1}(\bar{\Omega})$-minimizer of $\hat{\varphi}$. Invoking Proposition 2.7, we infer that $u_{*}$ is a local $W_{0}^{1, p}(\Omega)$-minimizer of $\widehat{\varphi}$. Similarly for $v_{*} \in-\operatorname{int} C_{+}$.

Without any loss of generality, we may assume that $\widehat{\varphi}\left(v_{*}\right) \leq \widehat{\varphi}\left(u_{*}\right)$ (the analysis is similar if the opposite inequality holds). Since $u_{*} \in \operatorname{int} C_{+}$is a local minimizer of $\widehat{\varphi}$, we can find $\rho \in(0,1)$ small such that

$$
\widehat{\varphi}\left(v_{*}\right) \leq \widehat{\varphi}\left(u_{*}\right)<\inf \left\{\widehat{\varphi}(u):\left\|u-u_{*}\right\|=\rho\right\}=\widehat{\eta}_{\rho}, \quad\left\|v_{*}-u_{*}\right\|>\rho .
$$


Recall that $\widehat{\varphi}$ is coercive (see 3.22)), hence it satisfies the PS-condition. This fact and (3.23) permit the use of Theorem 2.1 (the mountain pass theorem). So, we can find $y_{0} \in W_{0}^{1, p}(\Omega)$ such that

$$
\begin{gathered}
\widehat{\varphi}^{\prime}\left(y_{0}\right)=0 \quad \text { and } \quad \widehat{\eta}_{\rho} \leq \widehat{\varphi}\left(y_{0}\right), \\
\widehat{\varphi}\left(y_{0}\right)=\inf _{\gamma \in \Gamma} \max _{0 \leq t \leq 1} \widehat{\varphi}(\gamma(t)),
\end{gathered}
$$

where $\Gamma=\left\{\gamma \in C\left([0,1], W_{0}^{1, p}(\Omega)\right): \gamma(0)=v_{*}, \gamma(1)=u_{*}\right\}$. Since $y_{0} \in K_{\widehat{\varphi}} \subseteq$ $\left[v_{*}, u_{*}\right]$, it follows from (3.22) that $y_{0}$ is a solution of (1.1) and $y_{0} \in C_{0}^{1}(\bar{\Omega})$ (nonlinear regularity, see [17], [18]). If we show that $y_{0}$ is nontrivial, then the extremality of $u_{*}, v_{*}$ and (3.25) imply that $y_{0}$ is nodal. To show the nontriviality of $y_{0}$ we use (3.26). According to (3.26), in order to show that $y_{0}$ is nonzero, it suffices to produce a path $\gamma_{*} \in \Gamma$ such that $\left.\hat{\varphi}\right|_{\gamma_{*}}<0$. To this end, let

$$
\begin{aligned}
M & =W_{0}^{1, \tau}(\Omega) \cap \partial B_{1}^{L^{\tau}} & & \text { endowed with the relative } W_{0}^{1, p}(\Omega) \text {-topology, } \\
M_{c} & =M \cap C_{0}^{1}(\bar{\Omega}) & & \text { endowed with the relative } C_{0}^{1}(\bar{\Omega}) \text {-topology. }
\end{aligned}
$$

Recall that $\partial B_{1}^{L^{\tau}}=\left\{u \in L^{\tau}(\Omega):\|u\|_{\tau}=1\right\}$. Clearly, $M_{c}$ is dense in $M$. We introduce the following two sets of paths

$$
\begin{aligned}
\Gamma & =\left\{\widehat{\gamma} \in C([-1,1], M): \widehat{\gamma}(-1)=-\widehat{u}_{1}(\tau), \widehat{\gamma}(1)=\widehat{u}_{1}(\tau)\right\}, \\
\Gamma_{c} & =\left\{\widehat{\gamma} \in C\left([-1,1], M_{c}\right): \widehat{\gamma}(-1)=-\widehat{u}_{1}(\tau), \widehat{\gamma}(1)=\widehat{u}_{1}(\tau)\right\} .
\end{aligned}
$$

Claim 2. $\widehat{\Gamma}_{c}$ is dense in $\widehat{\Gamma}$.

Let $\widehat{\gamma} \in \widehat{\Gamma}$ and $\varepsilon \in(0,1)$. We introduce the multi-function $L_{\varepsilon}:[-1,1] \rightarrow$ $2^{C_{0}^{1}(\bar{\Omega})}$ defined by

$$
\begin{aligned}
L_{\varepsilon}(t) & =\left\{u \in C_{0}^{1}(\bar{\Omega}):\|u-\widehat{\gamma}(t)\|<\varepsilon\right\} \quad \text { for all } t \in(-1,1) ; \\
L_{\varepsilon}(-1) & =\left\{-\widehat{u}_{1}(\tau)\right\}, \quad L_{\varepsilon}(1)=\left\{\widehat{u}_{1}(\tau)\right\} .
\end{aligned}
$$

Clearly $L_{\varepsilon}$ has nonempty and convex values. Note that for $t \in(-1,1), L_{\varepsilon}(t)$ is open, while both $L_{\varepsilon}(-1)$ and $L_{\varepsilon}(1)$ are finite dimensional. Therefore $L_{\varepsilon}$ has values in the family $\mathcal{D}\left(C_{0}^{1}(\bar{\Omega})\right)$ of Michael [21]. Moreover, the continuity of $\widehat{\gamma}$ implies that the multifunction $L_{\varepsilon}$ is lower semi-continuous (see Papageorgiou amd Kyritsi [23, p. 458]). So, we can apply Theorem 3.1"1' of Michael [21] and produce a continuous map $\widehat{\gamma}^{\varepsilon}:[-1,1] \rightarrow C_{0}^{1}(\bar{\Omega})$ such that $\widehat{\gamma}^{\varepsilon}(t) \in L_{\varepsilon}(t)$ for all $t \in[-1,1]$. Let $\varepsilon_{n}=\frac{1}{n}$ and $\left\{\widehat{\gamma}^{n}=\widehat{\gamma}^{\varepsilon_{n}}\right\}_{n \geq 1} \subseteq C\left([-1,1], C_{0}^{1}(\bar{\Omega})\right)$ be the selectors produced above. Then

$$
\begin{gathered}
\left\|\widehat{\gamma}^{n}(t)-\widehat{\gamma}(t)\right\|<\frac{1}{n} \text { for all } t \in(-1,1), \\
\widehat{\gamma}^{n}(-1)=-\widehat{u}_{1}(\tau) \quad \text { and } \quad \widehat{\gamma}^{n}(1)=\widehat{u}_{1}(\tau) \quad \text { for all } n \geq 1 .
\end{gathered}
$$


Recall that $\widehat{\gamma}(t) \in \partial B_{1}^{L^{\tau}}$ for all $t \in[-1,1]$. So from (3.27) we see that we may assume that $\left\|\widehat{\gamma}^{n}(t)\right\| \neq 0$ for all $t \in[-1,1]$. Then we set

$$
\widehat{\gamma}_{0}^{n}(t)=\frac{\widehat{\gamma}^{n}(t)}{\left\|\widehat{\gamma}^{n}(t)\right\|_{\tau}} \quad \text { for all } t \in[-1,1] \text {. }
$$

Then $\widehat{\gamma}_{0}^{n} \in C\left([-1,1], M_{c}\right)$ and $\widehat{\gamma}_{0}(-1)=-\widehat{u}_{1}(\tau), \widehat{\gamma}_{0}(1)=\widehat{u}_{1}(\tau)$. Also, for all $t \in[-1,1]$ and all $n \geq 1$,

$$
\begin{aligned}
\left\|\widehat{\gamma}_{0}^{n}(t)-\widehat{\gamma}(t)\right\| & \leq\left\|\widehat{\gamma}_{0}^{n}(t)-\widehat{\gamma}^{n}(t)\right\|+\left\|\widehat{\gamma}^{n}(t)-\widehat{\gamma}(t)\right\| \\
& =\frac{\left|1-\left\|\widehat{\gamma}^{n}(t)\right\|_{\tau}\right|}{\left\|\widehat{\gamma}^{n}(t)\right\|_{\tau}}\left\|\widehat{\gamma}^{n}(t)\right\|+\frac{1}{n}
\end{aligned}
$$

(see (3.27)). Note that

$$
\begin{array}{rlrl}
\max _{-1 \leq t \leq 1}\left|1-\left\|\widehat{\gamma}^{n}(t)\right\|_{\tau}\right| & =\max _{-1 \leq t \leq 1}\left|\|\widehat{\gamma}(t)\|_{\tau}-\left\|\widehat{\gamma}^{n}(t)\right\|_{\tau}\right| & & \text { (recall that } \left.\widehat{\gamma}(t) \in \partial B_{1}^{L^{\tau}}\right) \\
& \leq \max _{-1 \leq t \leq 1}\left\|\widehat{\gamma}(t)-\widehat{\gamma}^{n}(t)\right\|_{\tau} & \\
& \leq C_{8} \max _{-1 \leq t \leq 1}\left\|\widehat{\gamma}(t)-\widehat{\gamma}^{n}(t)\right\| & & \text { for some } C_{8}>0 \\
& \leq \frac{C_{8}}{n} & & (\text { see }(3.19)) \\
\Rightarrow \max _{-1 \leq t \leq 1}\left\|\widehat{\gamma}_{0}^{n}(t)-\widehat{\gamma}(t)\right\| \rightarrow 0 & & \text { as } n \rightarrow \infty \\
\Rightarrow \widehat{\Gamma}_{C} \text { is dense in } \widehat{\Gamma} . &
\end{array}
$$

This proves Claim 2.

Using Claim 2 and Proposition 2.9, we see that given $\widehat{\delta}>0$, we can find $\widehat{\gamma}_{0} \in \widehat{\Gamma}_{c}$ such that

$$
\max _{-1 \leq t \leq 1}\left\|D \widehat{\gamma}_{0}(t)\right\|_{\tau}^{\tau} \leq \widehat{\lambda}_{2}(\tau)+\widehat{\delta}
$$

Also, from hypothesis $\left(\mathrm{H}_{1}^{\prime}\right)$ (iii) we have

$$
F(z, x) \geq \frac{c_{6}}{\tau}|x|^{\tau} \quad \text { for a.a. } z \in \Omega \text { and all }|x| \leq \delta_{0} .
$$

Moreover, hypothesis $\mathrm{H}(\mathrm{a})(\mathrm{iv})$ implies that given $\varepsilon>0$, we can find $\delta=\delta(\varepsilon)>0$ such that

$$
G(y) \leq \frac{\widetilde{c}+\varepsilon}{\tau}\|y\|^{\tau} \text { for all }\|y\| \leq \delta .
$$

Let $\delta_{1}=\min \{\widehat{\delta}, \delta\}$ (see (3.30) and (3.31)). Since $\widehat{\gamma}_{0} \in \widehat{\Gamma}_{c}$ and $u_{*} \in \operatorname{int} C_{+}$, $v_{*} \in-\operatorname{int} C_{+}$, we can find $\vartheta \in(0,1)$ small such that for all $t \in[-1,1]$ we have

$$
\vartheta \widehat{\gamma}_{0}(t) \in\left[v_{*}, u_{*}\right], \vartheta\left|\widehat{\gamma}_{0}(t)(z)\right|, \vartheta|| D \widehat{\gamma}_{0}(t)(z) \| \leq \delta_{1} \quad \text { for all } z \in \bar{\Omega} .
$$

Thus, for all $t \in[-1,1]$,

$$
\widehat{\varphi}\left(\vartheta \widehat{\gamma}_{0}(t)\right)=\int_{\Omega} G\left(\vartheta D \widehat{\gamma}_{0}(t)\right) d z-\int_{\Omega} F\left(z, \vartheta \widehat{\gamma}_{0}(t)\right) d z
$$


(see $(3.22),(3.32))$

$$
\leq \frac{\widetilde{c}+\varepsilon}{\tau} \vartheta^{\tau}\left\|D \widehat{\gamma}_{0}(t)\right\|_{\tau}^{\tau}-\frac{C_{6}}{\tau}\left\|\widehat{\gamma}_{0}(t)\right\|_{\tau}^{\tau}
$$

(see (3.30), (3.31), (3.32))

$$
\leq \frac{\vartheta^{\tau}}{\tau}\left[(\widetilde{c}+\varepsilon)\left(\widehat{\lambda}_{2}(\tau)+\widehat{\delta}\right)-C_{6}\right] .
$$

Since $c_{6}>\widetilde{c} \widehat{\lambda}_{2}(\tau)$ (see $\left.\left(\mathrm{H}_{1}^{\prime}\right)(\mathrm{iii})\right)$ by choosing $\varepsilon>0$ and $\widehat{\delta}>0$ small, relation (3.33) yields

$$
\widehat{\varphi}\left(\vartheta \widehat{\gamma}_{0}\right)(t)<0 \quad \text { for all } t \in[-1,1] .
$$

Therefore, if $\widehat{\gamma}=\vartheta \widehat{\gamma}_{0}$, then $\widehat{\gamma}$ is a continuous path in the Sobolev space $W_{0}^{1, p}(\Omega)$ which connects $-\vartheta \widehat{u}_{1}(\tau)$ and $\vartheta \widehat{u}_{1}(\tau)$, and

$$
\left.\widehat{\varphi}\right|_{\widehat{\gamma}}<0 \text {. }
$$

Next, we produce a continuous path in $W_{0}^{1, p}(\Omega)$ connecting $\vartheta \widehat{u}_{1}(r)$ and $u_{*}$ and along this path the functional $\hat{\varphi}$ is strictly negative. To this end, let

$$
a=\widehat{\varphi}_{+}\left(u_{*}\right)=\inf \left\{\widehat{\varphi}_{+}(u): u \in W_{0}^{1, p}(\Omega)\right\}<0=\widehat{\varphi}_{+}(0)
$$

(see the first part of the proof).

Applying the second deformation theorem (see, for example, Gasinski andPapageorgiou [13, p. 628]), we find a continuous map $h:[0,1] \times\left(\widehat{\varphi}_{+}^{0} \backslash K_{\widehat{\varphi}_{+}}^{0}\right) \rightarrow \widehat{\varphi}_{+}^{0}$ such that

$$
\begin{gathered}
h(0, u)=u \quad \text { for all } u \in \widehat{\varphi}_{+}^{0} \backslash K_{\hat{\varphi}_{+}}^{0}, \\
h\left(1, \widehat{\varphi}_{+}^{0} \backslash K_{\hat{\varphi}_{+}}^{0}\right) \subseteq \widehat{\varphi}_{+}^{a}
\end{gathered}
$$

and, for all $t, s \in[0,1], s \leq t$ and all $u \in \widehat{\varphi}_{+}^{0} \backslash K_{\hat{\varphi}_{+}}^{0}$,

$$
\widehat{\varphi}_{+}(h(t, u)) \leq \widehat{\varphi}_{+}(h(s, u)),
$$

From (3.23) we have $K_{\widehat{\varphi}_{+}}=\left\{0, u_{*}\right\}$. Hence $\widehat{\varphi}_{+}^{a}=\left\{u_{*}\right\}$ (see (3.35)). We deduce that

$$
\begin{aligned}
\widehat{\varphi}_{+}\left(\vartheta \widehat{u}_{1}(\tau)\right)=\widehat{\varphi}\left(\vartheta \widehat{u}_{1}(\tau)\right)=\widehat{\varphi}(\widehat{\gamma}(1))<0 & (\text { see }(3.34)) \\
& \Rightarrow \vartheta \widehat{u}_{1}(\tau) \in \widehat{\varphi}_{+}^{0} \backslash K_{\hat{\varphi}_{+}}^{0}=\widehat{\varphi}_{+}^{0} \backslash\{0\} .
\end{aligned}
$$

Hence we can define

$$
\widehat{\gamma}_{+}(t)=h\left(t, \vartheta \widehat{u}_{1}(\tau)\right)^{+} \text {for all } t \in[0,1] .
$$

Clearly, this defines a continuous path in $W_{0}^{1, p}(\Omega)$ and we have

$$
\begin{aligned}
\widehat{\gamma}_{+}(0) & =h\left(0, \vartheta \widehat{u}_{1}(\tau)\right)^{+}=\vartheta \widehat{u}_{1}(\tau) & & (\text { see }(3.36)) ; \\
\widehat{\gamma}_{+}(1) & =h\left(1, \vartheta \widehat{u}_{1}(\tau)\right)^{+}=u_{*} & & (\text { see }(3.37)) ; \\
\widehat{\varphi}\left(\widehat{\gamma}_{+}(t)\right) & =\widehat{\varphi}_{+}\left(\widehat{\gamma}_{+}(t)\right) \leq \widehat{\varphi}_{+}\left(\vartheta \widehat{u}_{1}(\tau)\right)=\widehat{\varphi}\left(\vartheta \widehat{u}_{1}(\tau)\right)<0 & & (\text { see }(3.38) \text { and }(3.34)) .
\end{aligned}
$$


Therefore $\widehat{\gamma}_{+}$is a continuous path in the Sobolev space $W_{0}^{1, p}(\Omega)$ which connects $\vartheta \widehat{u}_{1}(\tau)$ and $u_{*}$ and

$$
\left.\widehat{\varphi}\right|_{\widehat{\gamma}_{+}}<0 \text {. }
$$

In a similar fashion we produce $\widehat{\gamma}_{-}$a continuous path in $W_{0}^{1, p}(\Omega)$ which connects $-\vartheta \widehat{u}_{1}(\tau)$ and $v_{*}$ such that

$$
\left.\widehat{\varphi}\right|_{\hat{\gamma}_{-}}<0 .
$$

We concatenate the paths $\widehat{\gamma}_{-}, \widehat{\gamma}, \widehat{\gamma}_{+}$and produce a path $\gamma_{*} \in \Gamma$ such that

$$
\begin{aligned}
\left.\hat{\varphi}\right|_{\gamma_{*}} & <0 \quad(\text { see }(3.34),(3.39),(3.40)) \\
& \Rightarrow y_{0} \in C_{0}^{1}(\bar{\Omega}) \backslash\{0\} \text { is a nodal solution of (1.1). }
\end{aligned}
$$

So, we can conclude this section with the following multiplicity theorem for problem (1.1).

ThEOREM 3.8. Assume that hypotheses $\mathrm{H}(\mathrm{a})$ and $\left(\mathrm{H}_{1}^{\prime}\right)$ hold. Then problem (1.1) admits at least three nontrivial solutions $u_{0} \in \operatorname{int} C_{+}, v_{0} \in-\operatorname{int} C_{+}$, and $y_{0} \in\left[v_{0}, u_{0}\right] \cap C_{0}^{1}(\bar{\Omega})$ nodal.

\section{The $(p, 2)$-problem}

In this section we deal with the particular case of problem (1.1) in which $a(y)=\|y\|^{p-2} y+y$ for all $y \in \mathbb{R}^{N}$, with $2 \leq p<\infty$. Thus, the nonlinear nonhomogeneous Dirichlet problem under consideration is the following:

$$
-\Delta_{p} u(z)-\Delta u(z)=f(z, u(z)) \quad \text { in } \Omega,\left.\quad u\right|_{\partial \Omega}=0 .
$$

We have

$$
\nabla a(y)=\|y\|^{p-2}\left[I+(p-2) \frac{y \otimes y}{\|y\|^{2}}\right]+I
$$

for all $y \in \mathbb{R}^{N} \backslash\{0\}, \nabla a(0)=0$.

For this problem, if on the reaction term $f(z, x)$ we impose the stronger hypotheses $H_{2}$, we can localize more precisely the nodal solution $y_{0}$.

In what follows, $u_{*} \in \operatorname{int} C_{+}$and $v_{*} \in-\operatorname{int} C_{+}$are the extremal constant sign solutions from Proposition 3.6.

Proposition 4.1. Assume that hypotheses $\left(\mathrm{H}_{2}\right)$ hold. Then problem (4.1) has a nodal solution $y_{0} \in C_{0}^{1}(\bar{\Omega})$ such that $y_{0} \in \operatorname{int}_{C_{0}^{1}(\bar{\Omega})}\left[v_{*}, u_{*}\right]$.

Proof. From Proposition 3.7, we already have the existence of a nodal solution $y_{0} \in\left[v_{*}, u_{*}\right] \cap C_{0}^{1}(\bar{\Omega})$.

From (4.2) we see that $\nabla a\left(u_{*}(z)\right)$ and $\nabla a\left(v_{*}(z)\right)$ are both positive definite in $\Omega$. Hence we can apply the tangency principle of Pucci \& Serrin [27, p. 35]. Therefore

$$
v_{*}(z)<y_{0}(z)<u_{*}(z) \text { for all } z \in \Omega
$$


Let $\rho=\max \left\{\left\|v_{*}\right\|_{\infty},\left\|u_{*}\right\|_{\infty}\right\}$ and let $\xi_{\rho}>0$ be as postulated by hypothesis $\left(\mathrm{H}_{2}\right)(\mathrm{iv})$. Let $\widehat{\xi}_{\rho}>\xi_{\rho}$. Then

$$
\begin{aligned}
-\Delta_{p} y_{0}(z) & -\Delta y_{0}(z)+\widehat{\xi}_{\rho}\left|y_{0}(z)\right|^{p-2} y_{0}(z) \\
& =f\left(z, y_{0}(z)\right)+\widehat{\xi}_{\rho}\left|y_{0}(z)\right|^{p-2} y_{0}(z) \\
& =f\left(z, y_{0}(z)\right)+\xi_{\rho}\left|y_{0}(z)\right|^{p-2} y_{0}(z)+\left(\widehat{\xi}_{\rho}-\xi_{\rho}\right)\left|y_{0}(z)\right|^{p-2} y_{0}(z) \\
& \leq f\left(z, u_{*}(z)\right)+\xi_{\rho} u_{*}(z)^{p-1}+\left(\widehat{\xi}_{\rho}-\xi_{\rho}\right) u_{*}(z)^{p-1} \\
& =-\Delta_{p} u_{*}(z)-\Delta u_{*}(z)+\widehat{\xi}_{\rho} u_{*}(z)^{p-1}
\end{aligned}
$$

almost everywhere in $\Omega$, hence $u_{*}-y_{0} \in \operatorname{int} C_{+}$(see Proposition 2.8). In a similar fashion we show that $y_{0}-v_{*} \in \operatorname{int} C_{+}$. Therefore we conclude that $y_{0} \in \operatorname{int}_{C_{0}^{1}(\bar{\Omega})}\left[v_{*}, u_{*}\right]$.

Using this result and improving the regularity of $f(z, \cdot)$, we can produce a second nodal solution for problem (4.1) (for a total of four nontrivial smooth solutions). The new hypotheses on $f(z, x)$ are the following:

$\left(\mathrm{H}_{3}\right) f: \Omega \times \mathbb{R} \rightarrow \mathbb{R}$ is a measurable function such that for almost all $z \in \Omega$, $f(z, 0)=0, f(z, \cdot) \in C^{1}(\mathbb{R})$ and

(i) $\left|f_{x}^{\prime}(z, x)\right| \leq a(z)\left(1+|x|^{r-2}\right)$ for almost all $z \in \Omega$ and all $x \in \mathbb{R}$ with $a \in L^{\infty}(\Omega)_{+}, p \leq r<p^{*}$

(ii) $\lim _{x \rightarrow \pm \infty} f(z, x) /\left(|x|^{p-2} x\right)=-\infty$ uniformly for almost all $z \in \Omega$;

(iii) there exists an integer $m \geq 2$ such that $\widehat{\lambda}_{m}(2) \leq f_{x}^{\prime}(z, 0) \leq \widehat{\lambda}_{m+1}(2)$ almost everywhere in $\Omega, f_{x}^{\prime}(\cdot, 0) \neq \widehat{\lambda}_{m}(2), f_{x}^{\prime}(\cdot, 0) \neq \widehat{\lambda}_{m+1}(2)$, $f_{x}^{\prime}(z, 0)=\lim _{x \rightarrow 0} f(z, x) / x$ uniformly for almost all $z \in \Omega$.

REMARK 4.2. Hypotheses $\left(\mathrm{H}_{3}\right)$ (i), (iii) imply that for every $\rho>0$ we can find $\xi_{\rho}>0$ such that

$$
\begin{array}{ll}
f_{x}^{\prime}(z, x)+\xi_{\rho}(p-1)|x|^{p-2} \geq 0 & \text { for a.a. } z \in \Omega \text { and all }|x| \leq \rho \\
\Rightarrow \frac{\partial}{\partial x}\left[f(z, x)+\xi_{\rho}|x|^{p-2} x\right] \geq 0 & \text { for a.a. } z \in \Omega \text { and all }|x| \leq \rho \\
\Rightarrow x \mapsto f(z, x)+\xi_{\rho}|x|^{p-2} x & \text { is nondecreasing on }[-\rho, \rho] .
\end{array}
$$

Thus, hypotheses $H_{2}(i v)$ is automatically satisfied due to the stronger regularity of $f(z, \cdot)$.

Under these stronger conditions on the reaction $f(z, x)$ we can prove the following multiplicity theorem.

Theorem 4.3. Assume that hypotheses $\left(\mathrm{H}_{3}\right)$ hold. Then problem (4.1) admits at least four nontrivial solutions $u_{0} \in \operatorname{int} C_{+}, v_{0} \in-\operatorname{int} C_{+}$, and $y_{0}, \widehat{y} \in$ $\operatorname{int}_{C_{0}^{1}(\bar{\Omega})}\left[v_{0}, u_{0}\right]$ nodal. 
Proof. From Theorem 3.8 and Proposition 4.1, we already have three solutions $u_{0} \in \operatorname{int} C_{+}, v_{0} \in-\operatorname{int} C_{+}$and $y_{0} \in \operatorname{int}_{C_{0}^{1}(\bar{\Omega})}\left[v_{0}, u_{0}\right]$ nodal.

Without any loss of generality we may assume that $u_{0}, v_{0}$ we are extremal (that is, $u_{0}=u_{*}, v_{0}=v_{*}$ ). Using the notation introduced in Proposition 3.7, we obtain that $u_{0} \in \operatorname{int} C_{+}$and $v_{0} \in-\operatorname{int} C_{+}$are both local minimizer of $\widehat{\varphi}$. Hence

$$
C_{k}\left(\widehat{\varphi}, u_{0}\right)=C_{k}\left(\widehat{\varphi}, v_{0}\right)=\delta_{k, 0} \mathbb{Z} \text { for all } k \geq 0 .
$$

Since $y_{0} \in \operatorname{int}_{C_{0}^{1}(\bar{\Omega})}\left[v_{0}, u_{0}\right]$ and $\left.\varphi\right|_{\left[v_{0}, u_{0}\right]}=\left.\widehat{\varphi}\right|_{\left[v_{0}, u_{0}\right]}$, we have

$$
\begin{aligned}
C_{k}\left(\left.\varphi\right|_{C_{0}^{1}(\bar{\Omega})}, y_{0}\right)=C_{k}\left(\left.\widehat{\varphi}\right|_{C_{0}^{1}(\bar{\Omega})}, y_{0}\right) \quad \text { for all } k & \geq 0 \\
\Rightarrow C_{k}\left(\varphi, y_{0}\right) & =C_{k}\left(\widehat{\varphi}, y_{0}\right) \quad \text { for all } k \geq 0
\end{aligned}
$$

(see Palais [22] and Bartsch [4]). From the proof of Proposition 3.7, we know that $y_{0}$ is a critical point of $\widehat{\varphi}$. Hence

$$
C_{1}\left(\widehat{\varphi}, y_{0}\right) \neq 0 \Rightarrow C_{1}\left(\varphi, y_{0}\right) \neq 0
$$

(see (4.4)). Since $\varphi \in C^{2}\left(W_{0}^{1, p}(\Omega)\right)$, using (4.5) as in Papageorgiou and Smyrlis [26] (see the proof of Proposition 13), we obtain for all $k \geq 0$

$$
C_{k}\left(\varphi, y_{0}\right)=\delta_{k, 1} \mathbb{Z} \Rightarrow C_{k}\left(\widehat{\varphi}, y_{0}\right)=\delta_{k, 1} \mathbb{Z}
$$

Also, by virtue of hypothesis $\left(\mathrm{H}_{3}\right)(\mathrm{iii})$, from Papageorgiou and Smyrlis [26, Proposition 10] we have

$$
C_{k}(\widehat{\varphi}, 0)=\delta_{k, d_{m}} \mathbb{Z} \quad \text { for all } k \geq 0, \quad \text { with } d_{m}=\operatorname{dim} \bigoplus_{i=1}^{m} E\left(\widehat{\lambda}_{i}(2)\right) .
$$

Finally, recall that $\widehat{\varphi}$ is coercive (see $(3.22)$ ). Hence

$$
C_{k}(\widehat{\varphi}, \infty)=\delta_{k, 0} \mathbb{Z} \quad \text { for all } k \geq 0 .
$$

Suppose that $K_{\widehat{\varphi}}=\left\{0, u_{0}, v_{0}, y_{0}\right\}$. Then from (4.3), (4.6), (4.7), (4.8) and the Morse relation with $t=-1$ (see $(2.5)$ ), we have $(-1)^{d_{m}}+2(-1)^{0}+(-1)^{1}=(-1)^{0}$, a contradiction. So, we can find $\widehat{y} \in K_{\widehat{\varphi}}, \widehat{y} \notin\left\{0, u_{0}, v_{0}, y_{0}\right\}$. Since $\widehat{y} \in\left[v_{0}, u_{0}\right]$ (see $(3.23)$ ), we conclude that $\widehat{y} \in C_{0}^{1}(\bar{\Omega})$ is nodal. Moreover, as in the proof of Proposition 4.1, we show that $\widehat{y} \in \operatorname{int}_{C_{0}^{1}(\bar{\Omega})}\left[v_{0}, u_{0}\right]$.

\section{Parametric equations}

In this section we deal with the following parametric $p$-Laplacian Dirichlet equation

(5.1) $-\Delta_{p} u(z)=\lambda|u(z)|^{p-2} u(z)-f(z, u(z)) \quad$ in $\Omega,\left.\quad u\right|_{\partial \Omega}=0,1<p<\infty$.

Here, $\lambda>0$ is a parameter. The hypotheses on the perturbation term $f(z, x)$ are the following:

$\left(\mathrm{H}_{4}\right) f: \Omega \times \mathbb{R} \rightarrow \mathbb{R}$ is a Carathéodory function such that $f(z, 0)=0$ for almost all $z \in \Omega$ and 
(i) for every $\rho>0$, there exists $a_{\rho} \in L^{\infty}(\Omega)_{+}$such that $|f(z, x)| \leq$ $a_{\rho}(z)$ for almost all $z \in \Omega$ and all $|x| \leq \rho$;

(ii) $\lim _{x \rightarrow \pm \infty} f(z, x) /\left(|x|^{p-2} x\right)=+\infty$ uniformly for almost all $z \in \Omega$;

(iii) $\lim _{x \rightarrow 0} f(z, x) /\left(|x|^{p-2} x\right)=0$ uniformly for almost all $z \in \Omega$.

For problem (5.1), $a(y)=\|y\|^{p-2} y$ for all $y \in \mathbb{R}^{N}, \tau=p$ and $\widetilde{c}=1$ (see hypothesis $\mathrm{H}(\mathrm{a})(\mathrm{iv}))$. By virtue of hypothesis $\left(\mathrm{H}_{4}\right)(\mathrm{iii})$, we see that given $\varepsilon>0$, we can find $\delta_{0}=\delta_{0}(\varepsilon)>0$ such that

$$
f(z, x) x \leq \varepsilon|x|^{p} \quad \text { for a.a. } z \in \Omega \text { and all }|x| \leq \delta_{0} .
$$

Hence we have

$$
\lambda|x|^{p}-f(z, x) x \geq(\lambda-\varepsilon)|x|^{p} \quad \text { for a.a. } z \in \Omega \text { and all }|x| \leq \delta_{0} .
$$

If $\lambda>\widehat{\lambda}_{2}(p)$, then we choose $\varepsilon \in\left(0, \lambda-\widehat{\lambda}_{2}(p)\right)$ and we can apply Theorem 2.7. Thus, we obtain

TheOREM 5.1. Assume that hypotheses $\left(\mathrm{H}_{3}\right)$ hold and $\lambda>\widehat{\lambda}_{2}(p)$. Then problem (5.1) has at least three nontrivial solutions $u_{0} \in \operatorname{int} C_{+}, v_{0} \in-\operatorname{int} C_{+}$, and $y_{0} \in\left[v_{0}, u_{0}\right] \cap C_{0}^{1}(\bar{\Omega})$ nodal.

REMARK 5.2. Such a multiplicity result was first proved by Ambrosetti and Mancini [2], with subsequent improvements by Ambrosetti and Lupo [1] and Struwe [29], [30, p. 132], when $p=2$ and $f(z, \cdot)=f(\cdot) \in C^{1}(\mathbb{R})$ or even Lipschitz continuous. In their multiplicity theorems, they do not show that the third solution is nodal. This result was extended to $p$-Laplacian equations by Papageorgiou and Papageorgiou [24]. Again, it is not shown that the third solution is nodal.

In the semilinear case $(p=2)$, with a more regular reaction $f(z, \cdot)$ and with additional restrictions on the parameter $\lambda>0$, we can improve Theorem 5.1 and produce a second nodal solution. So, we consider the following semilinear parametric problem:

$$
-\Delta u(z)=\lambda u(z)-f(z, u(z)) \quad \text { in } \Omega,\left.\quad u\right|_{\partial \Omega}=0 .
$$

The hypotheses on the perturbation $f(z, x)$ are the following:

$\left(\mathrm{H}_{4}\right) f: \Omega \times \mathbb{R} \rightarrow \mathbb{R}$ is a measurablefunction such that for almost all $z \in \Omega$, $f(z, 0)=0, f(z, \cdot) \in C^{1}(\mathbb{R})$ and

(i) $\left|f_{x}^{\prime}(z, x)\right| \leq a(z)\left(1+|x|^{r-2}\right)$ for almost all $z \in \Omega$ and all $x \in \mathbb{R}$, with $a \in L^{\infty}(\Omega)_{+}, 2 \leq r<2^{*}$

(ii) $\lim _{x \rightarrow \pm \infty} f(z, x) / x=+\infty$ uniformly for almost all $z \in \Omega$;

(iii) $\lim _{x \rightarrow 0} f(z, x) / x=0$ uniformly for almost all $z \in \Omega$. 
In what follows, $\widehat{\sigma}(2)$ denotes the spectrum of $\left(-\Delta, H_{0}^{1}(\Omega)\right)$, that is, $\widehat{\sigma}(2)=$ $\left\{\widehat{\lambda}_{k}(2)\right\}_{k \geq 1}$ (see Section 2). Using Theorem 4.3, we obtain the following multiplicity property.

THEOREM 5.3. Assume that hypotheses $\left(\mathrm{H}_{4}\right)$ hold and $\lambda>\widehat{\lambda}_{2}(2), \lambda \notin \widehat{\sigma}(2)$. Then problem (5.3) has at least four nontrivial solutions $u_{0} \in \operatorname{int} C_{+}, v_{0} \in$ $-\operatorname{int} C_{+}$, and $y_{0}, \widehat{y} \in \operatorname{int}_{C_{0}^{1}(\bar{\Omega})}\left[v_{0}, u_{0}\right]$ nodal.

\section{REFERENCES}

[1] A. Ambrosetti And D. Lupo, One class of nonlinear Dirichlet problems with multiple solutions, Nonlinear Anal. 8 (1984), 1145-1150.

[2] A. Ambrosetti And G. Mancini, Sharp nonuniqueness results for some nonlinear problems, Nonlinear Anal. 3 (1979), 635-645.

[3] D. Arcoya And D. Ruiz, The Ambrosetti-Prodi problem for the p-Laplacian operator, Comm. Partial Differential Equations 31 (2006), 849-865.

[4] T. Bartsch, Critical point theory on partially ordered Hilbert spaces, J. Funct. Anal. 186 (2001), 117-152.

[5] V. Benci, P. D’Avenia, D. Fortunato and L. Pisani, Solutions in several space dimensions: Derrick's problem and infinitely many solutions, Arch. Ration. Mech. Anal. 154 (2000), 297-324.

[6] H. Brezis and L. Nirenberg, $H^{1}$ versus $C^{1}$ local minimizers, CRAS Paris 317 (1993), $465-472$.

[7] L. Cherfils AND Y. IlyAsov, On the stationary solutions of generalized reaction diffusion equations with p\&q-Laplacian, Comm. Pure Appl. Anal 4 (2005), 9-22.

[8] M. Cuesta, G. De Figueiredo And J.-P. Gossez, The beginning of the Fučik spectrum for the p-Laplacian, J. Differential Equations 159 (1999), 212-238.

[9] J.I. DIAZ AND J.E. SAA, Existence et unicité de solutions positives pour certaines équations elliptiques quasilinéaires, C.R. Acad. Sci. Paris Sér. I Math. 305 (1987), 521524.

[10] N. Dunford and J. Schwartz, Linear Operators, Vol. I, Wiley-Interscience, New York, 1958.

[11] M. Filippakis, A. Kristaly and N.S. Papageorgiou, Existence of five nonzero solutions with exact sign for a p-Laplacian equation, Discrete Cont. Dynam. Systems 24 (2009), 405-440.

[12] J.P. Garcia Azorero, J. Manfredi And I. Peral Alonso, Sobolev versus Hölder local minimizers and global multiplicity for some quasilinear elliptic equations, Comm. Contemp. Math. 2 (2000), 385-404.

[13] L. Gasinski And N.S. Papageorgiou, Nonlinear Analysis, Chapman \& Hall/CRC, Bocan Raton, 2006.

[14] _ Multiple solutions for nonlinear coercive problems with a nonhomogeneous differential operator and a nonsmooth potential, Set-Valued Var. Anal. 20 (2012), 417-443.

[15] Z. GUO AND Z. Zhang, $W^{1, p}$ versus $C^{1}$ local minimizers and multiplicity results for quasilinear elliptic equations, J. Math. Anal. Appl. 286 (2003), 32-50.

[16] A. Kristaly, V. Rădulescu and C. Varga, Variational Principles in Mathematical Physics, Geometry and Economics: Qualitative Analysis of Nonlinear Equations and Unilateral Problems, Cambridge Univ. Press, Cambridge, 2010. 
[17] O.A. Ladyzhenskaya And N. URAL'TSEva, Linear and Quasilinear Elliptic Equations, Academic Press, New York, 1968.

[18] G.M. Lieberman, The natural generalization of the natural conditions of Ladyzhenskaya and Ural'tseva for elliptic equations, Comm. Partial Differential Equations 16 (1991), 311-361.

[19] S. Liu, Multiple solutions for coercive p-Laplacian equations, J. Math. Anal. Appl. 316 (2006), 229-236.

[20] J. LiU AND S. LiU, The existence of multiple solutions to quasilinear elliptic equations, Bull. London Math. Soc. 37 (2005), 592-600.

[21] E. Michael, Continuous selections I, Ann. Math. 63 (1956), 361-382.

[22] R. PALAis, Homotopy theory of infinite dimensional manifolds, Topology 5 (1966), 1-16.

[23] N.S. Papageorgiou and S. Kyritsi, Handbook of Applied Analysis, Springer, New York, 2009.

[24] E. Papageorgiou and N.S. Papageorgiou, A multiplicity theorem for problems with the p-Laplacian, J. Funct. Anal. 244 (2007), 63-77.

[25] N.S. Papageorgiou And V.D. RĂdulescu, Qualitative phenomena for some classes of quasilinear equations with multiple resonance, Appl. Math. Optim. 69 (2014), 393-430.

[26] N.S. Papageorgiou And G. Smyrlis, On nonlinear nonhomogebeous resonant Dirichlet equations, Pacific J. Math. 264 (2013), 421-453.

[27] P. Pucci and J. Serrin, The Maximum Principle, Birkhäuser, Basel, 2007.

[28] V.D. Rădulescu, Qualitative Analysis of Nonlinear Partial Differential Equations, Hindawi Publ. Co., New York, 2008.

[29] M. Struwe, A note on a result of Ambrosetti and Mancini, Ann. Mat. Pura Appl. 131 (1982), 107-115.

[30] _ Variational Methods, Springer, Berlin, 1990.

[31] J.-L. VAzquez, A strong maximum principle for some quasilinear ellpitic equations, Appl. Math. Optim 12 (1984), 191-202.

Manuscript received October 4, 2013

Nikolaos S. Papageorgiou

National Technical University, Department of Mathematics

Zografou Campus

Athens 15780, GREECE

E-mail address: npapg@math.ntua.gr

Vicenţiu D. RĂDUlescu

Department of Mathematics Faculty of Science

King Abdulaziz University

Jeddah, SAUDI ARABIA

and

Institute of Mathematics

"Simon Stoilow" of the Romanian Academy

P.O. Box 1-764

014700 Bucharest, ROMANIA

E-mail address: vicentiu.radulescu@math.cnrs.fr

TMNA : Volume $45-2015-\mathrm{N}^{\mathrm{O}} 2$ 